\title{
DEE/ER/B2565-1
}

\section{Phase II Final Report}

\section{DOE Grant \#: DE FG02-98ER82565}

Topic Area: Safeguards, Security, and Material Accountability for SNM

\author{
DOE Project Officer: Carl Friesen \\ Contracting Officer: Chris Swierczek \\ Administered for DOE by: \\ Earlette Robinson, DOE/ACQ \\ 9800 South Cass Ave., \\ Argonne, IL 60439
}

Recipient: Dr. F. David Doty, PI

Doty Scientific, Inc.

700 Clemson Rd.

Columbia, SC 29223

(803) $788-6497$

Project Title:

\section{Development of 1-10 W, 10 K Reverse Brayton Cycle Cryocoolers}

F. D. Doty, Art Boman, Sam Arnold, J. B. Spitzmesser, Doug Jones, D. L. McCree, L. J. Hacker

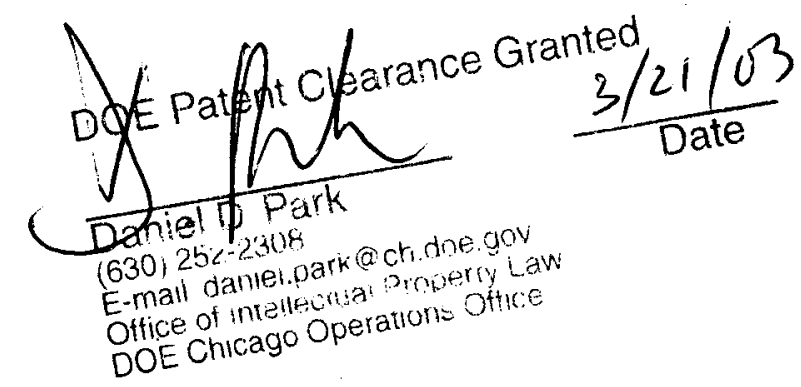




\section{DISCLAIMER}

This report was prepared as an account of work sponsored by an agency of the United States Government. Neither the United States Government nor any agency Thereof, nor any of their employees, makes any warranty, express or implied, or assumes any legal liability or responsibility for the accuracy, completeness, or usefulness of any information, apparatus, product, or process disclosed, or represents that its use would not infringe privately owned rights. Reference herein to any specific commercial product, process, or service by trade name, trademark, manufacturer, or otherwise does not necessarily constitute or imply its endorsement, recommendation, or favoring by the United States Government or any agency thereof. The views and opinions of authors expressed herein do not necessarily state or reflect those of the United States Government or any agency thereof. 


\section{DISCLAIMER}

Portions of this document may be illegible in electronic image products. Images are produced from the best available original document. 


\section{Development of 1-10 W, 10 K Reverse Brayton Cycle Cryocoolers \\ Phase II Final Report \\ PI: F. David Doty}

\section{Contents}

1. Significance of the Problem, and General Technical Approach 3

1.1 The Microturbine Expanders 3

1.1.1 Crash-tolerant, Ultra-low-power, High-speed, Gas Bearings 4

1.1.2 Application of the NMR MAS Spinner to the Miniature RBC Cryocooler 5

$\begin{array}{lll}1.2 & \text { The Micro-generator } & 7\end{array}$

1.3 Compact Recuperators $\quad 8$

1.4 The Compressor 10

1.5 The Low-Pressure Reverse Brayton Cycle 11

2. Summary of Phase II Objecticves 14

3. Phase II Efforts and Results 15

$\begin{array}{lll}3.1 & \text { The Microturbine Expanders } & 17\end{array}$

3.2 Computational Fluid Dynamics (CFD) 23

3.2.1 CFD Software Selection $\quad 23$

3.2.2 Simulations on Microturbine Expanders $\quad 24$

3.2.3 Simulations on the Compressor $\quad 28$

$\begin{array}{lll}3.3 & \text { The Micro-generator } & 31\end{array}$

3.4 Compact Recuperators $\quad 31$

3.4.1 Manufacture $\quad 32$

3.4.2 Compact Shell-side Manifolding to Improve Homogeneity of flow $\quad 37$

3.4.3 Tube-side Homogeneity and Subchannel Coupling 39

$\begin{array}{lll}3.5 & \text { Overall System } & 41\end{array}$

4. Update of Initial Phase III Efforts $\quad$. 43

4.1 Compact Recuperators 43

4.2 Micro-turbine Expanders $\quad 45$

5.- References 46 


\section{Significance of the Problem, and General Technical Approach}

Miniature cryocoolers for the 8-30 $\mathrm{K}$ range are needed to provide $0.5-5 \mathrm{~W}$ of cooling to high-sensitivity detectors (for long-wave-length IR, magnetism, mm-wave, X-ray, dark matter, and possibly $\gamma$-ray detection) while maintaining low mass, ultra-low vibration, and good efficiency. For example, HgCdTe mid-IR (3-5 $\mu \mathrm{m}) 256 \times 256$ focal plane arrays (40x40 $\mu \mathrm{m}$ pixels) in CCD cameras, obtain spectral detectivities of $\sim 2 \times 10^{9} \mathrm{cmHz}{ }^{1 / 2} \mathrm{~W}$ at $5 \mathrm{kHz}$ when operating at $145 \mathrm{~K}$ (Freon 14 b.p.), and nearly an order of magnitude improvement is achieved at $77 \mathrm{~K}$. Obtaining high-speed detectivities near quantum limits in the 6-14 $\mu \mathrm{m}$ long-IR range with $1024 \times 1024$ arrays of 8-15 $\mu \mathrm{m}$ pixels will require cooling to $8-30 \mathrm{~K}$, and vibration amplitudes must be below a small fraction of the pixel size. Available products are still inadequate for these and many other vibration-sensitive fieldable sensor applications.

This project presents a new approach to eliminating the problems normally encountered in efforts to build low-vibration, fieldable, miniature cryocoolers. Using the Reverse Brayton Cycle $(\mathrm{RBC})$, the approach applies and expands on existing spinner technology previously used only in Nuclear-Magnetic Resonance (NMR) probes.

We have focused on the RBC because of several fundamentally appealing attributes of this cycle which have been proven in mid- and large-scale systems [5]: (1) The vibration amplitude at the cold head (we measured $0.1 \mu \mathrm{m}$ ) is at least two orders of magnitude lower than that of viable alternatives below $20 \mathrm{~K}$ (Stirling or Gifford McMahon) without active cancellation and in a frequency range more easily isolated; (2) it may approach the Carnot efficiency limit (typically within a factor of three to five); (3) very high reliability and long mean-time-betweenmaintenance (MTBM) have been achieved in many mid- and large-scale systems; (4) it may be readily adapted to any temperature down to $\sim 8 \mathrm{~K}$; and (5) low specific mass may be achieved.

To date, these benefits have not been realized in low-power cryocoolers - due to specific technical problems in microturbines, micro-generator loads, high-effectiveness compact recuperators, and high-speed gas bearings. For each of these specific limiting factors to RBC low-power cryocoolers, significant progress has been made during this Phase II. The RBC system is discussed in more detail in section 1.5 after discussions of each critical component and our general technical approach to it. The initially proposed cryocooler system flow diagram is shown in Figure 1, and an RBC T-S diagram is shown in Figure 4, page 11.

1.1 The Microturbine Expanders. The miniature RBC cooler remains a challenging goal. The show-stopper in the past has generally been either reliability or suitability of the micro-turbine expanders' gas bearings. The expanders themselves need to be very small yet able to attain high surface velocities. The first problem repeatedly encountered is that unavoidable crashes (touch-downs) at high speed occur from resonances, blockages, severe acceleration, power failures, and routine shut-down. Trying to design a simple control system that prevents crashes in the microturbine expander has proven unsuccessful, and typical gasbearing turbine expanders survive fewer than three crashes without major damage to the 
bearings [6]. The second problem commonly plaguing previous gas bearing designs is that they have introduced unacceptable losses (either viscous, convective, or conductive) usually setting the lower power limit at 50-400 $\mathrm{W}$ of cooling at $20-80 \mathrm{~K}[7,8]$. We have learned new lessons during the Phase II about microturbine bearing frictional losses and the extreme scale-down necessary to achieve low such low power at low $<30 \mathrm{~K}$.

\subsubsection{Crash-tolerant, Ultra-low-power,} High-speed, Gas Bearings. Doty Scientific has acquired extensive experience with efficient microturbines driving NMR Magic Angle Spinning (MAS) sample spinners on gas bearings at rotational frequencies up to $27 \mathrm{kHz}$ (1.6 million $\mathrm{rpm}$ ) over the past two decades [Doty 12]. These silicon-nitride sample spinners are used in hundreds of laboratories around the world and experience moderately high-speed $(\sim 200 \mathrm{~m} / \mathrm{s})$ crashes many times per day (every time the sample is changed) with virtually no damage. They are currently in routine use at surface speeds up to $345 \mathrm{~m} / \mathrm{s}$. With well balanced samples, peak vibration amplitude of the stator for our $5 \mathrm{~mm}$ rotor appears to be under 0.3 microns, and the stator mass is only $0.013 \mathrm{~kg}$.

The most important factor in crashtolerant bearings is all-silicon-nitride

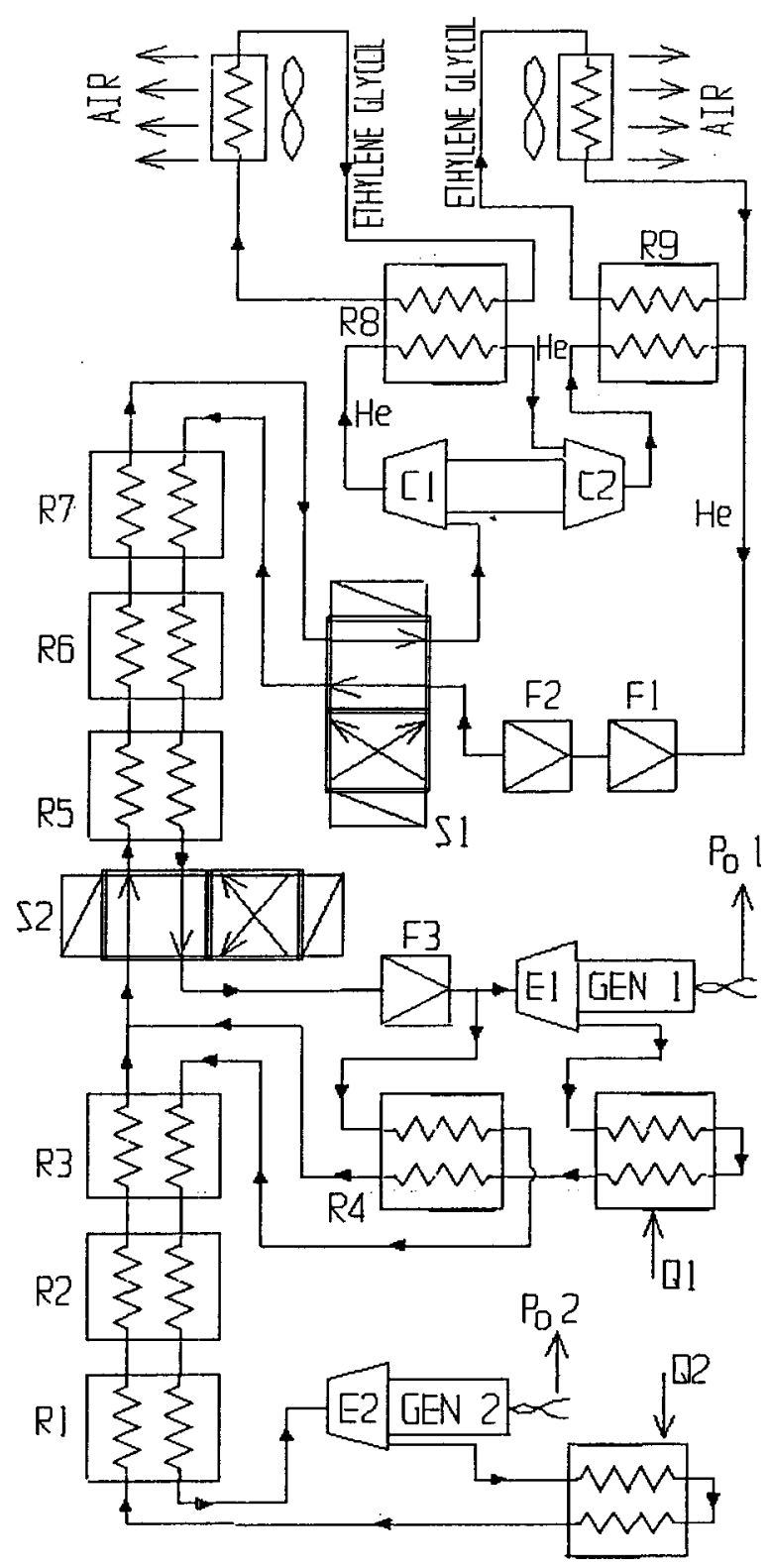

Figure 1. Flow Schematic for the Initially Proposed 2-stage LP-RBC construction. Silicon nitride's exceptional wear resistance arises from its high microhardness at $1400 \mathrm{~K}$ (the peak surface temperatures generated in a severe crash), high thermal conductivity, high fracture toughness, low thermal expansion, and chemical inertness. An additional important factor in crash tolerance is the ratio of bearing surface area to stored inertial energy. This ratio is roughly proportional to $\left(\omega^{2} \rho d^{3}\right)^{-1}$, where $\omega_{\mathrm{r}}$ is the rotational rate, $\rho$ is the rotor density, and $d$ is the rotor diameter. Hence, the low density of silicon nitride and small diameter of the rotors also contribute to their robustness.

From the classic expression for the onset of Taylor vortices and the desire to maximize bearing stiffness but minimize friction and bearing mass flow rate, it can be shown that optimum bearing radial clearance $r_{c}$ is given by an expression of the following form [Doty 11]: 


$$
r_{C} \cong k T^{0.55} r^{1 / 3}
$$

where $T$ is the bearing temperature and $r$ is the bearing radius. For helium gas and (MAS) typical aspect ratios, the coefficient $k$ has value $\sim 0.0013 \mathrm{~K}^{-0.55} \mathrm{~mm}^{2 / 3}$. For nitrogen, it is about $60 \%$ of that amount. For most MAS applications, where thermal gradients are to be minimized at sonic surface speeds, the bearing hole size is such that the mass flow with the rotor in place is about $85 \%$ of that with the rotor removed. For the RBC expander bearing, where total power loss is to be minimized and mass flow is more critical, the above optimum flow ratio is reduced.

We have developed a simple method (that is very effective) for controlling the "whirl instability" which has often plagued other gas bearing designs $[8,13]$. The half-frequency whirl instability is caused by the phase shift between the shaft displacement and the hydrostatic restoring force of the gas bearing that arises from the mean rotational velocity of the gas in the bearing clearance - nominally being half the rotor rotational rate for radially aligned bearing holes. The key to controlling whirl instabilities is simply inclining the bearing holes radially at about $25^{\circ}$ so as to oppose the direction of shaft rotation [Doty 11]. We denote this as the "antiwhirl supersonic bearing", since bearing surface speeds over $345 \mathrm{~m} / \mathrm{s}(\sim 6 \%$ above the sonic velocity) have been demonstrated with air at $\sim 350 \mathrm{~K}$.

1.1.2 Application of the NMR MAS Spinner to the Miniature RBC Cryocooler. We have shown in numerous experiments that the combination of generator loading, axial thrust balance, pure radial inflow expander turbines, partial admission, reduced bearing diameters, and optimized anti-whirl ceramic bearings provide the opportunity for two orders of magnitude reduction in cooling power at a practical efficiency. However, recent experiments show greater drop in efficiency than expected in microturbines as the admission ratio drops below $\sim 0.3$. Hence, it will be necessary to use smaller microturbines than initially expected to obtain adequate efficiency, and this entails substantial development and major design revisions. We realized late in the final Phase 11 period that a 2-mm-OD spinner bearing was required to achieve the low leakage and bearing pressure necessary.

To simplify the design of an efficient, single-stage microturbine (and because of our continued advances in compact, high-effectiveness heat exchangers - a development which reduces the benefit of higher pressure ratios), we expect to operate at a turbine pressure ratio $r$ of 2.5. Our prototype microturbines have demonstrated $37 \%$ efficiency at $300 \mathrm{~K}$ for air flow under $0.7 \mathrm{~g} / \mathrm{s}$ (blade Reynolds numbers under 3500 , shaft power $\sim 11 \mathrm{~W}$ ). Higher efficiency is expected for optimized and precisely machined microturbines in helium at $10 \mathrm{~K}$ at $\sim 2 \mathrm{~W}$ shaft power, as Reynolds numbers will be nearly an order of magnitude higher and the sonic velocity is considerably lower.

As previously noted, the bearing for an RBC microturbine must generate minimal heat load. Most prior RBC designs have loaded the expander turbine with a room-temperature (RT) compressor. This does not appear to be a viable option below $\sim 50 \mathrm{~W}$ for two reasons: (1) it is not easy to make the conductive heat leak to the RT compressor less than $\sim 12 \mathrm{~W}$, and (2) the heat load and momentum losses associated with leakage from the large gas bearing generally exceed $\sim 8 \mathrm{~W}$. 
The bearing leakage is largely controlled simply by operating with very small radial clearances $(6-12 \mu \mathrm{m})$ as indicated by eq. [1]. Unfortunately, a tight bearing has high frictional losses and is not stable during the cool-down. A compromise clearance, optimized for $\sim 50 \mathrm{~K}$, has been shown to work adequately for some purposes over the full temperature range [Doty 12]. However, another approach that could be considered for the cryocooler is to provide separate manifolding for several subsets of the bearing holes and drive nozzles. The bearing and drive flows may then be changed with cold flow-switches as the temperature changes.

So the problem of unacceptable shaft losses can only be addressed with an integral generator load inside the coldhead. Several simple generator designs have proven effective for rotors of this size. With the entire stator and load at the inlet temperature, conductive losses from the RBC turboexpander may be virtually eliminated. Our analysis suggested that convective and viscous bearing losses could be kept under $0.15 \mathrm{~W}$ using a $2 \mathrm{~mm}$ gas bearing at $7 \mathrm{kHz}$ with $3 \mathrm{~mm}$ partial-admittance microturbines.

The requirement of an efficient generator within a few millimeters of the bearing and turbine necessitates all-ceramic construction, as eddy current losses in metals would degrade generator efficiency unacceptably.

A simple and exceptionally efficient generator design, previously used at room temperature and compatible with all-ceramic construction, was shown to be suitable for use at temperatures from 4-400 K: a transversely magnetized cylinder of $\mathrm{Nd}-\mathrm{B}-\mathrm{Fe}$ is secured inside the silicon nitride bearing rotor and a simple quadrupolar winding on a low-loss core is placed around the bearing stator. More comments will be made later on generator issues.

While radial gas bearings are now easy to stabilize, our experience with thrust microbearings of all types and sizes has convinced us that it is unrealistic to expect them to perform adequately over a wide range of conditions unless very conservatively loaded. The only practical solution in microturbine applications is to design with axial symmetry that eliminates axial loads as much as possible. This suggests that below a certain size, microturbines must be mounted in balanced pairs. (This is one of those places where making two is easier than making one).

Small turbine designs prior to our pioneering work in this area used essentially isotropic reductions of designs (mixed radialaxial-inflow, etc.) that had worked well in the several kilowatt range. The problem with extending this approach below $\sim 100 \mathrm{~W}$ is that the small diameter requires rotational rates that exceed even the capabilities of our anti-

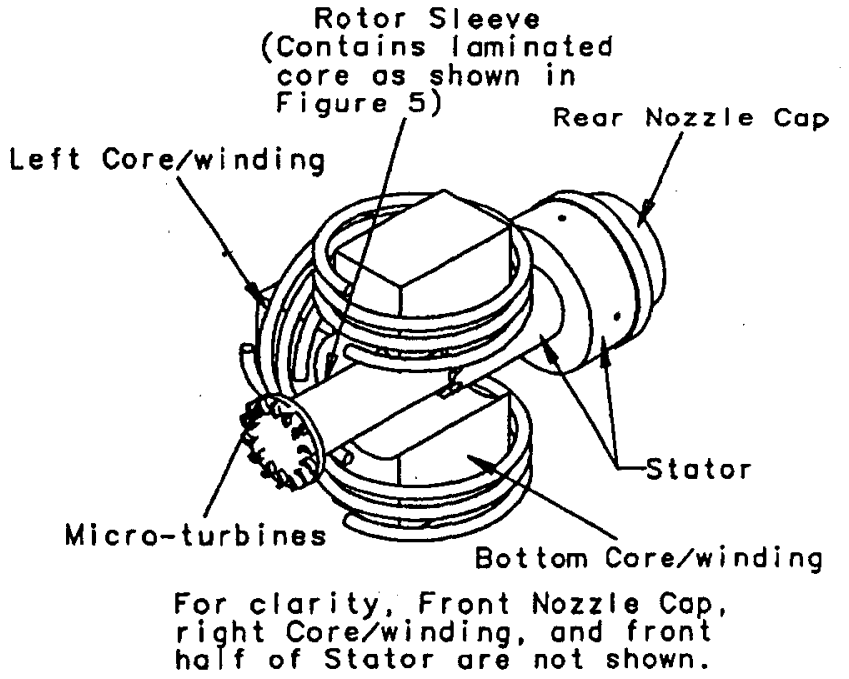

Figure 2. The preliminary RBC micro-expandergenerator design concept 
whirl bearings with efficient generator loads. Previous small turbine designs have paid close attention to minimizing surface area in the turbine, but total bearing and shaft surface area and bearing surface speed are actually more important in minimizing losses in microturbines. Hence, the turbine diameter in efficient RBC micro-expanders must exceed the bearing diameter. Preliminary experiments suggest this will not lead to conical resonance problems in our latestgeneration expander/bearing design, even under conditions far from the design operating range.

High-efficiency, single-stage turbines must operate with tip speeds at over half the speed of sound. Because of micro-manufacturing constraints, the best approach is a radial-inflow design that allows the axial blade dimension at inlet to be reduced to a fraction of a millimeter while maintaining a reasonable turbine diameter. With the axial blade length very short at the perimeter, extremely small flow areas at high tip speeds are achieved without excessive rotational frequencies.

While we have shown that partial admission can perform acceptably for some microturbine applications, we have concluded, contrary to our initial expectations, that full admission is the only route possible for the efficiency needed in the cryocooler expander. The reason is not that the turbine itself could not be made to have acceptable efficiency with partial admission, but that the only way to reduce the gas bearing losses to an acceptable level is to reduce their size and hence the diameter of the turbine. (Engineers not familiar with gas bearings may find it surprising that total bearing losses may be greatly reduced by using a smaller bearing at a higher rotational rate, even though frictional power is quadratic with rotational rate.) However, the emphasis should still be on low reaction ratio, as this minimizes tip leakage and rotational rate.

Our latest CFD simulations show that a practical $3 \mathrm{~mm}$ expander turbine at $50 \mathrm{~K}$ (with 2 $\mathrm{mm}$ gas bearing diameter) should be able to achieve over $55 \%$ polytropic efficiency at a mass flow of $\sim 0.3 \mathrm{~g} / \mathrm{s}$. More details of the RBC turbine expander work are discussed later.

1.2 The Micro-generator. The micro-generator (or alternator, in automotive parlance) design is as critical as the turbine design and was initially perceived to be of significant technical risk. Designing a micro-generator capable of efficient operation at speeds from $4 \mathrm{kHz}$ at $8 \mathrm{~K}$ to $25 \mathrm{kHz}$ at $300 \mathrm{~K}$ was not expected to be trivial - partially because the relatively large thickness of the rotor and bearing stator gives a high-reluctance field, which requires the use of a dipolar core [19] (higher orders decay too rapidly with distance). However, our recent microcryo-generator demonstrations were completely successful.

Of course, a number of other options were considered; and preliminary, parametric computer models were developed to assist in evaluating these options. An induction generator's efficiency with high-reluctance fields appeared to be too low (during initial cool-down) for $4 \mathrm{~mm}$ rotors above $60 \mathrm{~K}$ - even at $10 \mathrm{kHz}$.

The simple ferrite permanent-magnet cylindrical (PMC) generator - a rotating hard-ferrite cylindrical dipole with quadrature field windings - was also evaluated, as this permits high efficiency even at high frequency since there are no eddy current losses in the rotor core. The 
problem is that available hard ferrites (e.g., strontium ferrite) lose too much coercivity below 150 to $250 \mathrm{~K}$.

The switched-reluctance (or variable-reluctance) generator appeared to be promising for certain applications, and again a dipolar core and quadrupolar field were analyzed. The advantage of this design is that the highly stressed core material is neither a permanent magnet nor a good conductor, as the permanent magnets (or energized windings) are in the field [19, 20]. The soft magnetic component of the core may be either powdered iron or laminated Si-steel. The core filler material may be epoxy composite or ceramic. This may be the best approach for generator loads required to work in external fields between 0.3 and $1.5 T$ (as in MRI/NMR magnets), but that is not our concern at the moment.

The most promising option for use in external fields below $0.3 \mathrm{~T}$ appeared to be the PMC design with a neodymium-boron-iron core instead of a hard ferrite, though reliable data on its coercivity and remanence over the full temperature range were not available and other hard magnets were known to be unsuitable at low temperatures. Moreover, its electrical conductivity $\left(7.7 \times 10^{5} \mathrm{~S} / \mathrm{m}, \sim 1 \%\right.$ that of $\left.\mathrm{Cu}\right)$ was thought to be high enough to result in significant rotor core eddy current losses in a $2.8 \mathrm{~mm}$ solid core above $\sim 2 \mathrm{kHz}$. Also, with $\mathrm{Nd}-\mathrm{B}-\mathrm{Fe}$, the stresses could become problematic above $-3 \mathrm{kHz}$ owing to its high density and low strength. However, the possibility of high coercivity demanded our attention, and luck was with us. Nd-B-Fe was found to have very stable coercivity over the full temperature range with no significant degradation from repeated thermal cycling. Moreover, its mean transverse thermal expansion is (surprisingly) a very close match to that of silicon nitride. Also, the quadrature high-reluctance field windings virtually eliminate cogging (since $\cos ^{2} \theta+\sin ^{2} \theta=1$ ).

Just as surprising, preliminary experiments have shown that, with a low-reluctance quadrature field winding, eddy current losses may be kept low enough in a solid Nd-B-Fe rotor core to be of little significance at least up to $8 \mathrm{kHz}$. Although soft ferrites are not suitable below $\sim 150 \mathrm{~K}$ for the field core, low-perm iron-power field cores permit very high efficiency with a hyperconducting winding. The bottom line is that the high-speed cryo-generator design problem is now perceived to be rather easy.

1.3 Compact Recuperators. The recuperators needed for the RBC do not rely on thermal storage, so they have no lower temperature limit. In-house micro-fabrication techniques permit compact gas-to-gas recuperators to have net effectiveness greater than $99 \%$ at temperatures down to $\sim 4.5 \mathrm{~K}$, where the ultra-low gas viscosity makes pumping losses acceptable even with channel diameters as small as $0.1 \mathrm{~mm}$ [Doty 15]. But we have discovered during Phase II that there is a better option for the low-temperature stage-II recuperator that relies on the hyper-conductivity of copper at very low temperatures. For the Stage I recuperators, substantial progress was made in compact recuperator development with several exciting beneifits for cryogenic and high-temperature applications. 
Our micro-tube strip (MTS) heat exchangers have permitted a factor of five increase in specific conductance $\left(S_{C}\right.$, heat-exchange power per mass per degree temperature difference, W/kgK) compared to prior compact designs with over $96 \%$ effectiveness [Doty 15]. The design is conceptually simple, as illustrated in Figure 3 . The key to the improvement is the unique
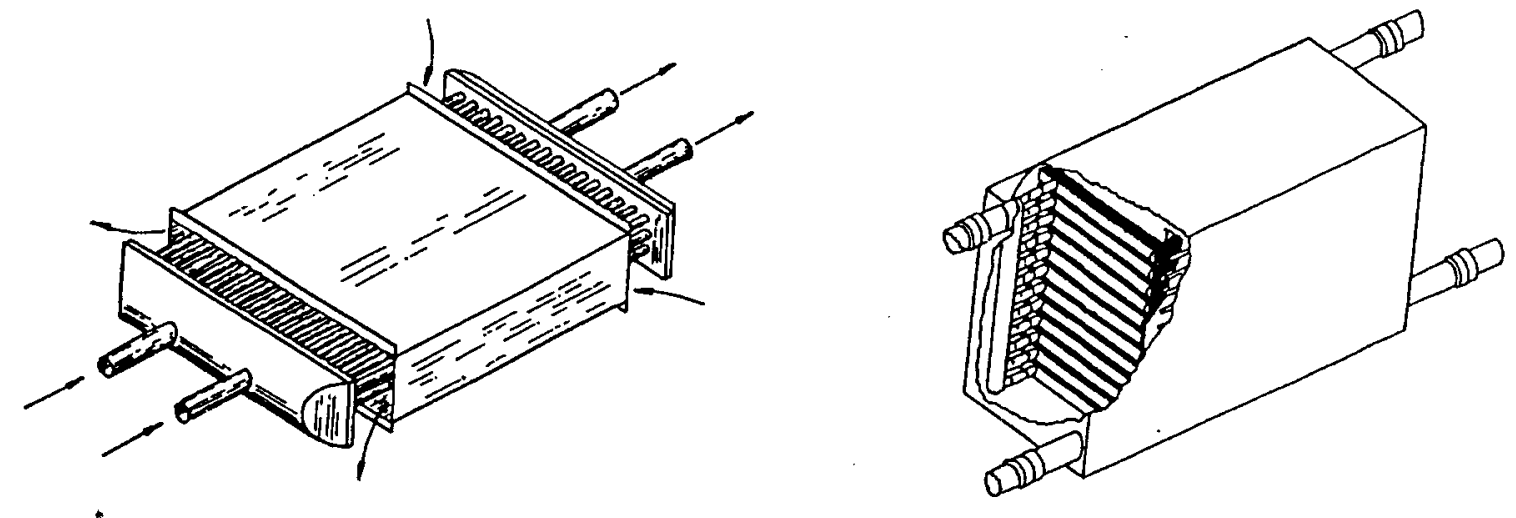

Figure 3. One MTS heat exchanger module and a bank of MTS modules.

manifolding of rectangular counterflow modules that permits excellent flow uniformity over an exceptionally large fraction of the tube length - even with over 1000 microtubes per module [Doty 22]. It arose from the realization that heat transfer power per unit mass under laminar counterflow conditions (which permit minimal pressure loss at a given effectiveness) is inversely proportional to the square of the tube diameter.

For high-effectiveness laminar exchangers, the minimum tube inside diameter $d_{\text {min }}$ is quite small and is given approximately by the following:

$$
d_{\min } \simeq 1000\left(k \mu T_{\delta}\right)^{1 / 2} /\left[\left(T_{H}-T_{C}\right) \rho C_{P}\right]
$$

where $k$ is the gas conductivity $(\mathrm{W} / \mathrm{mK}), \mu$ is the dynamic viscosity $(\mathrm{kg} / \mathrm{ms}), \rho$ is the gas density $\left(\mathrm{kg} / \mathrm{m}^{3}\right), T_{\delta}$ is the mean temperature difference between the counterflowing streams, $T_{H}$ is the highest temperature, $T_{\mathrm{C}}$ is the lowest temperature, and $C_{\mathrm{P}}$ is the gas specific heat. This amounts to $\sim 0.1 \mathrm{~mm}$ for helium exchangers at $20 \mathrm{~K}$ and $0.3 \mathrm{MPa}$, which is well below practical manufacturing limits. Microtubes may be produced at very low cost (under $\$ 0.15 / \mathrm{m}$ ) so automation should permit economical, ultra-compact exchangers if suitable brazing and manufacturing methods can be developed.

It appears that the minimum practical microtube outside diameter with our available manufacturing processes is about $0.65 \mathrm{~mm}$ for tube lengths of 200 to $400 \mathrm{~d}$, depending on flexural strength, when high effectiveness must be achieved. Silver-alloy brazing of low-conductivity Cu$\mathrm{Ni}$ alloy microtubes is much easier than alternative joining processes with stainless alloys, and both chemical and conventional machining methods of the header strips are less costly than fineblanking for small-scale production. Detailed analyses, both from a conventional and from a novel approach, have been published, and our detailed computer model of the MTS heat exchanger has been proven in both small and large applications. 
Flow inhomogeneity within a single module is not easily kept below $\sim 1.5 \%$. It arises primarily from the effects of inaccuracy in tube spacing (even with six to ten position-control grids) on shell-side flow distribution within the module and from the difficulty in keeping the shell-side flow cage spaced properly from the outer rows of tubes within each module. Thus, although the axial conduction loss is easily kept below $1 \%$, it is not easy to achieve more than $\sim 97 \%$ effectiveness in a single module unless more attention is paid to the shell-side flow distribution at the inlet and outlet than shown in Figure 3. We have developed a simple improvement for single modules (a non-uniform distribution of shell-side feed holes in a shell-side flow distributor strip at the inlet and outlet) to provide uniform inlet/outlet distributions with minimal increase in pressure drop over a wide range of flow conditions. Also, manifolding improvements made during Phase II have led to manufacturability of high-temperature recuperators being developed now as a separate project. More details on these latest features are discussed later.

When pressure drops are low (as is the case at low temperatures) the modules or banks of modutes may be connected in series with simple, turbulent mixers between units to greatly reduce the effects of flow maldistribution. For example, in a 2-stage RBC, three MTS exchanger banks in series for the recuperator between $300 \mathrm{~K}$ and $50 \mathrm{~K}$ could achieve the desired $98.6 \%$ effectiveness for the first stage, and three small modules in series between $50 \mathrm{~K}$ and $10 \mathrm{~K}$ should provide the required effectiveness in this range with little difficulty - though our research has shown that other options may be better here.

1.4 The Compressor. In the RBC, the compressor performance is generally less critical than the expander performance, as the expander performance has a more pronounced effect on recuperator size (which is much more expensive than the RT rejector) as well as a greater effect on coefficient of performance (COP). Since the compressor can be highly isolated from vibration sensitive instruments (except perhaps in space applications) and effective fluid filtration systems are available, it is often possible to utilize reciprocating compressors. Of course, turbo-compressors offer the potential for much lower mass, vibration, and contamination, but their efficiency has generally been unacceptable for helium at room temperature with mass flow rates below $-15 \mathrm{~g} / \mathrm{s}$. The progress we have demonstrated in gas bearings provides the basis for high-speed motors and bearings appropriate for helium turbo-compressors down to 2 $\mathrm{g} / \mathrm{s}$. However, the major and unexpected changes necessary to the expander prevented any Phase II compressor efforts beyond design and CFD optimizations.

Some of the best published results with "miniature" helium compressors have utilized regenerative (analogous to partial admittance expanders) axial flow units [26], as they permit reduced rotational rate for a given turbine OD. However, with gas bearings, it is more important to reduce the mass of the rotor than its rotational rate, as this more directly affects the critical conical resonance for a given bearing size. With crash-tolerant gas bearings, the rotational rate can be greatly increased. Replacing one regenerating blisk with a few centrifugal compressors in series should reduce the total blisk mass by $\sim 2$ orders of magnitude.

Somewhat lower efficiencies than initially expected are now being predicted by the CFD simulations, which means the compressor outlet temperatures and hence the outlet sonic 
velocities are increased. But more importantly, the original estimates also contained errors in materials properties. While the short-term yield strength of the titanium beta alloys remains very high up to $600 \mathrm{~K}$, the long-term rupture strength of these alloys declines very rapidly above 375 K. Even if $66 \%$ polytropic efficiency can be achieved, with two compressors in series with an intercooler, the outlet temperature of the second compressor will be $\sim 410 \mathrm{~K}$. The bottom line is that higher peripheral speeds are needed for a 2-stage design than can possibly be sustained by any available material under long-term operation. While it might be possible to achieve a system compression ratio of 2.2 (about the lowest practical from a recuperator-size perspective) from three compressors in series, it is much better to go with four compressors so that balanced pairs can be used to simplify the axial gas-bearing design problem. Now, even for a rather extreme compressor inlet temperature of $315 \mathrm{~K}$, the compressor outlet temperature is under $375 \mathrm{~K}$, where the high-strength titanium beta alloys can be used with excellent long-term performance.

While the ideal cycle efficiency would be improved (as there will now be three intercoolers instead of one), the compressor bearing gas requirement is doubled. Since this helium compressor load is essentially independent of refrigerent mass flow over a very wide range of values it makes sense to design for a total mass flow that is large compared to the bearing gas flow. Otherwise the final system would not compare favorably with competitive cryocoolers with respect to efficiency.
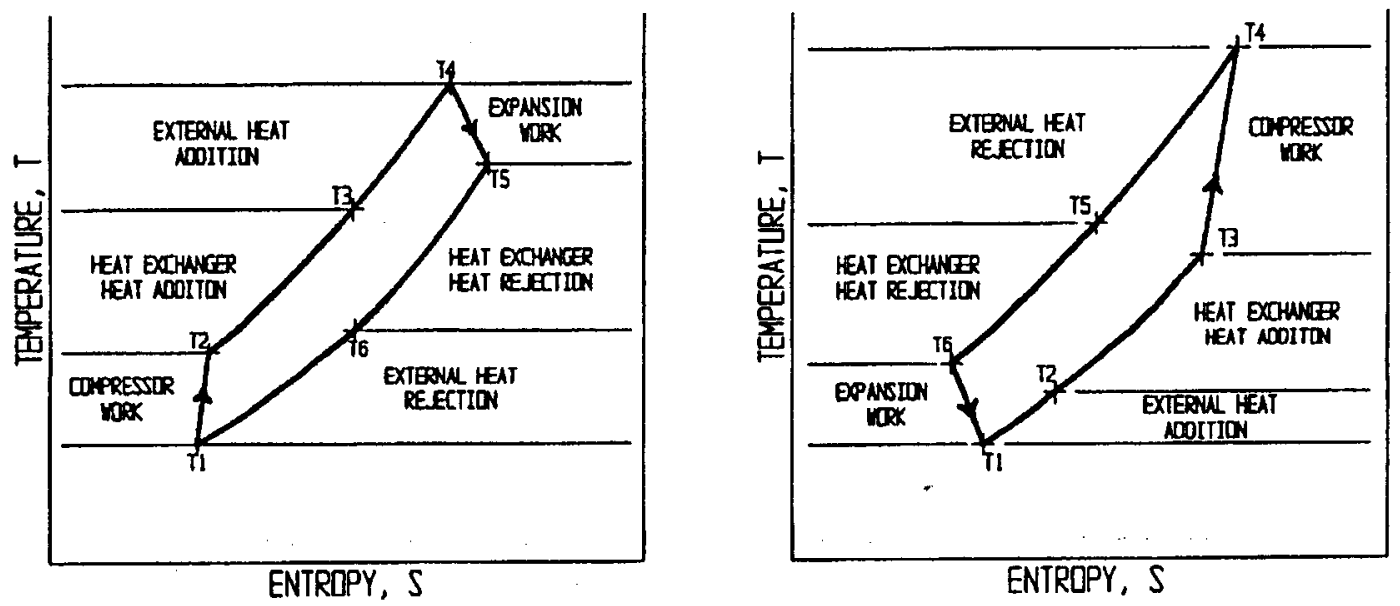

Figure 4. (a) The T-S diagram for the Closed Brayton (power) Cycle (CBC) is shown on the left, and (b) the single-stage Reverse Brayton (refrigeration) Cycle is shown on the right.

1.5 The Low-Pressure Reverse Brayton Cycle. The efficiency $\eta_{s}$ of the Closed Brayton (power) Cycle ( $C B C$ ), as illustrated in Figure $4 a$, with perfect gases $\left(C_{\mathrm{P}}\right.$ assumed to be independent of temperature) without intercool or interheat is given by the following $[18,27]$ :

$$
\eta_{S}=\frac{\eta_{H} \eta_{E}(E \tau-C)}{\tau\left[\eta_{X}(C+E)-C\right]}
$$


where $\eta_{E}$ is the generator efficiency, $\eta_{H}$ is the heater efficiency, $\eta_{X}$ is the recuperator effectiveness, $\tau=T_{4} / T_{1}, E=\left(T_{4}-T_{5}\right) / T_{4}$, and $C=\left(T_{2}-T_{1}\right) / T_{1}$. Assuming a perfect gas (which, with helium, is a valid approximation except in the expander below $\sim 15 \mathrm{~K}$ ), we can write:

$$
\begin{aligned}
& E=1-\left(r-\delta_{r}\right)^{(1-\gamma) \varepsilon / \gamma} \\
& C=r^{(\gamma-1) / \gamma \varphi}-1
\end{aligned}
$$

where $r$ is the compression ratio, $\varphi$ is the compressor polytropic efficiency, $\varepsilon$ is the expander polytropic efficiency, $\gamma$ is the ratio of specific heats $C_{P} / C_{V}$, and $\delta_{r}$ is the normalized pressure drop in the ducts and recuperator - i.e., $\Sigma \delta p / p$. The COP (heat lifted/work done) of the RBC refrigeration cycle (Fig. $4 b)$ is essentially $\left(\tau \eta_{S}\right)^{-1}$ except that $\eta_{E}$ remains in the numerator (for the cold-generator-loaded expander) and $\eta_{H}$ is replaced with $\eta_{M}$, the compressor motor efficiency. With bearing losses properly included in $\eta_{E}$ and $\eta_{M}$, the following may be written:

$$
C O P=\frac{\eta_{M} \eta_{E}\left[\eta_{X}(C+E)-C\right]}{(E \tau-C)}
$$

The refrigeration efficiency $\eta_{R}$, usually defined as follows, is often reported:

$$
\eta_{R}=\frac{C O P}{C O P_{\text {CARNOT }}}=\frac{\eta_{M} \eta_{E}\left[\eta_{X}(C+E)-C\right]\left(T_{5}-T_{1}\right)}{(E \tau-C) T_{1}}
$$

For $\eta_{\mathrm{X}}>\left(1-\left(r^{1 / 2}-1\right) / 4 \tau\right), r<(1+\tau), \delta_{\mathrm{r}}<(r-1) / 50$, and with bearing losses included in $\eta_{\mathrm{E}}$ and $\eta_{M}$, the following approximation is usually accurate within $\pm 30 \%$ :

$$
\eta_{R} \cong 0.7 \eta_{M} \eta_{E} \varepsilon \varphi
$$

From the above conditions for eq. [8] or from eq. [7], one finds that single-stage cryocoolers with low $r$ and large $\tau$ require extremely high $\eta_{x}$ (often above 0.995), which makes 2stage coolers much more practical.

There are two viable approaches to the 2-stage RBC: (A) Use approximately the same compression ratio as would be selected for a single stage, expand part of the gas at an intermediate temperature, and expand the rest of the gas at the second stage temperature; (B) Start at twice the compression ratio, expand all of the gas to an intermediate pressure at an intermediate temperature, and complete the expansion at the second stage temperature. The first technique at a relatively high pressure ratio (with the addition of a J-T bottoming cycle) is essentially the classic Samuel Collins helium liquefier. It is the technique reported herein, but 
with a low pressure ratio (so that a 4-stage helium turbo-compressor can be made to work well), along with several minor changes and additions. We'll simply denote it as the 2-stage LowPressure (LP) RBC.

With relatively minor modifications, the same turbo-compressor could also be able to perform well as a hydrogen compressor, which is likely to be of interest for a modified Collins-type hydrogen cycle for refrigeration down a few watts and up to much higher power in the temperature range of 22-28 K. Such a cryocooler could be considerably more compact (the MTS exchangers would be smaller) than the $10 \mathrm{~K} \mathrm{RBC}$ and less expensive, as the only expander turbine needed is for the first stage at $\sim 70 \mathrm{~K}$ and there are no leakage problems to deal with in the $\mathrm{J}-\mathrm{T}$ bottoming stage. 


\section{Summary of Phase II Objectives}

This section lists the original objectives of the Phase II project, as discussed in detail in the Phase II proposal, and Section 3 reports the actual Phase II effort and results:

2.1 Design, manufacture, and optimize $6 \mathrm{~mm}$ cryo-expanders with $4 \mathrm{~mm}$ bearings.

.1 Optimize bearing stability and efficiency for helium for the range $8 \mathrm{~K}$ to $90 \mathrm{~K}$.

.1.1 Determine whether or not dual bearing manifolds and flow switches are needed.

.1.2 Perform and verify numerical simulations of radial and axial bearings using CFD software.

.1.3 Evaluate eddy-current damping if axial instabilities arise.

.2 Optimize generator field pole material and contour for minimum hysteresis loss.

.2.1 Perform numerical simulations of the PMC quadrature generator.

.2.2 Improve and further develop parametric generator/motor design software.

.3 Optimize 1st-stage 6-mm turbine efficiency for helium for the $40-80 \mathrm{~K} \mathrm{range}, 0.3 \mathrm{~g} / \mathrm{s}$.

.3.1 Perform and verify numerical simulations of microturbines using CFD software.

.3.2 Improve and further develop parametric micro-turbine design software.

.4 Optimize 2 di-stage $6-\mathrm{mm}$ turbine efficiency for helium for the $10-20 \mathrm{~K}$ range, $0.3 \mathrm{~g} / \mathrm{s}$.

.4 .1 Design and test rapid-heating of stator for automatic cleaning.

.4 .2 Demonstrate $\eta_{E}$ greater than $35 \%$ at $\sim 3 \mathrm{kHz}, 1 \mathrm{~W}, 10 \mathrm{~K}$.

.5 Produce 3 each $(10 \mathrm{~K}, 50 \mathrm{~K})$ optimized expanders for system experiments and field tests.

2.2 Design, manufacture, and optimize $20 \mathrm{~mm}$ centrifugal compressor with $10 \mathrm{~mm}$ bearings.

.1 Optimize bearing designs (stability and efficiency) for both helium and air at $330 \mathrm{~K}$.

.2 Achieve $96 \%$ efficiency in $10 \mathrm{~mm}, 1-\mathrm{kW}, 6 \mathrm{kHz}$ AC motor.

.3 Perform and verify numerical simulations of centrifugal compressors using CFD software.

.4 Achieve $40 \%$ efficiency in air turbo-compressor for $r=4$ per stage, $2.5 \mathrm{~g} / \mathrm{s}$.

.5 Achieve $40 \%$ efficiency in helium turbo-compressor for $r=1.6$ per stage, $<0.8 \mathrm{~g} / \mathrm{s}$.

.6 Produce 3 optimized helium turbo-compressors with AC power supplies.

2.3 Detailed design, production, and testing of MTS recuperators.

.1 Demonstrate $>99 \%$ effectiveness for each stage, $315-50 \mathrm{~K}$ and $50-10 \mathrm{~K}$.

.2 Design, analyze, and produce 3 cryostats and manifolding.

2.4 Complete detailed design and manufacture of (3) $1 \mathrm{~W}, 10 \mathrm{~K}$ cryocoolers.

.1 Further develop RBC system optimization software.

.2 Design system control unit and develop control software.

.3 Perform manufacturing and subcontracting cost analyses.

2.5 Write Phase II Final Report.

2.6 Submit technical papers to appropriate journals. 


\section{Phase II Efforts and Results}

Doty Scientific, Inc. has put forth an extensive and efficient R\&D effort (including a significant in-kind contribution of capital and direct resources) during Phase II and made substantial progress. Numerous technical benefits in multiple areas have been realized, and progress and significant technical contributions continue in cryogenics and other fields as a result of the Phase II and ongoing efforts. Not withstanding this, many of the aggressive Phase II Proposal objectives were not met even though the Phase II effort was well planned.

Execution of the Phase II research path presented several large surprises beyond those expected and normally present in extremely high-tech development work. By far the largest unexpected set-back was the high expander-bearing leakage and friction; this necessitated six full generations of expanders. Although, the real problem was not the total iterations but the two major scale-downs that were needed. During Phase II, we went from a $5 \mathrm{~mm}$ bearing OD, to a 4 $\mathrm{mm}$ OD (even though at the time our standard NMR products only went to $5 \mathrm{~mm}$ ), and finally to design of a $2 \mathrm{~mm}$ expander - which represents a substantial quantum reduction in size to us and to the state-of-the art in small, high-speed spinners (see Section 4.2). Fortunately, a significant CFD investigation and optimization showed the necessity of the second scale-down prior to the end Phase II. This saved resources, and allowed them to be put toward $2 \mathrm{~mm}$ expander CFDwork, design, and materials. Following completion of this Phase II, $2 \mathrm{~mm}$ expander work for noncryogenic applications continues with in-kind and alternate-funding resources.

Although we still believe that a 1-10 W, $10 \mathrm{~K} \mathrm{RBC}$ cryocooler is feasible as described in this report (though with less certainty than it was believed at the end of Phase I), the development work required for a full system demonstration is at least double what it was believed to be even six months ago, as partially outlined in the previous paragraph. This has necessitated selection of project objectives to pursue from among the total body of project objectives outlined in the preceding section. Phase III commercialization has begun on a component level, but is not expected to occur on a cryogenic-system level without additional, funded, Phase-II work.

Phase I feasibility studies showed that the compact heat exchangers proposed were manufacturable, as the key MTS issue appeared to be manufacturability. This was based on our previous experience with these exchangers in early prototypes. Of course, there were some of the normal unanticipated problems with manufacturing during Phase II that were overcome. Once stage one MTS recuperators were fabricated, though, we experienced continued shell-side flow problems. Two generations of exchangers went through design, procurement, and manufacture. The stage II exchangers experienced similar problems for different reasons, and went through two generations as well. To sum up briefly, in the heat exchangers, it was found that shell-side flow inhomogeneities in the recuperator could not be reduced sufficiently without utilizing more deliberate efforts to distribute the shell-side flow uniformly across the exchanger module at inlet and outlet. A practical method of doing so was devised and tested with excellent results (see section 3.4.2). Even with these deliberate methods the stage II heat exchanger had inadequate performance. We finally decided that the problem there was caused by insufficient 
spacing between the tubes (the tube spacing error was becoming a significant fraction of the spacing). The results were a large subchannel (the flow areas between tubes on the shell side) hydraulic-diameter variation and insufficient subchannel cross-flow coupling to even out inhomogeneities (see section 3.4.3).

It is clear from the Phase II developments outlined in the above paragraphs that the original objectives in section 2 did no longer constitute an appropriate development path, as the resulting device would have been inadequate. Also, as outlined, unanticipated work necessitated prioritizing of project objectives. Thoughtful prioritizing allowed the Phase II to be a success and yield many beneficial innovations. Phase III work has begun and we expect that objective 2.6 above (contributions to the literature and the state of the art) will continue for some time. Throughout Phase II, we placed a high priority on development and optimization of a $2 \mathrm{~mm}$ expander for four reasons:

1. It's development is essential to the low power RBC.

2. We believed that few, if any, other organizations in the world could develop such an expander.

3. There are several spin-off technical benefits from the development work.

4. None of the large, originally-proposed capital investments (which would put an undue drain on resources relative to any DOE benefit) were required to perform this work.

Similar logic applies to the heat-exchanger development, although not item 2 above. Both of these components also appeared in the last year to be the most likely to reach Phase III (See section 4).

Specifically, In the $4 \mathrm{~mm}$ micro-expander, the experiments showed that (1) leakage from the drive plenum to the bearing exhaust and the effects of partial admission at the inlet flow had a more detrimental effect on turbine efficiency than our earlier experiments had predicted, and (2) viscous losses in the axial bearing and labyrinth seal were considerably greater than expected. The only apparent solution is to (1) reduce the turbine diameter to the point that a full-admission turbine can be made with sufficiently small flow rate and (2) reduce the gas bearing and generator core diameters for compatibility with the required increase in rotational rate (to $>2,000,000 \mathrm{rpm}$ ). Hence, (1) the turbine diameter must be reduced from $6 \mathrm{~mm}$ to $3 \mathrm{~mm},(2)$ the silicon-nitride gas bearing diameter must be reduced from $4 \mathrm{~mm}$ to $2 \mathrm{~mm}$, and (3) the generator magnet diameter drops from $2.9 \mathrm{~mm}$ to $1.4 \mathrm{~mm}$. Preliminary analysis of the generator re-design shows it will still have sufficient output power and efficiency. Detailed re-design of the scaleddown micro-expander and micro-generator is complete and CFD simulations of the required 3 $\mathrm{mm}$ microturbines for both the $50 \mathrm{~K}$ and the $10 \mathrm{~K}$ expander show that the not only will the reduced diameter greatly reduce bearing friction and drive-plenum leakage, it will also allow higher efficiency of the turbines themselves, as the span dimension will be increased. The factorof-two scale-down in the precision silicon-nitride micro-parts would not be trivial, but it appears feasible.

The CFD simulations of the compressor showed us that the outlet temperatures will be 
higher than expected, and we learned that the long-term rupture strength of the titanium beta alloys drops sharply above $375 \mathrm{~K}$. Even if $64 \%$ polytropic efficiency can be achieved in each stage, with two compressors in series with an intercooler the outlet temperature of the second compressor will be $\sim 410 \mathrm{~K}$. The bottom line is that higher tip speeds are needed for a 2-stage design than can possibly be sustained by any available material under long-term operation. We decided to go with four centrifugal compressors (with intercool) as this permits balanced pairs to be used to simplify the axial gas-bearing design problem. Most importantly, we discovered from our CFD simulations that water cooling the compressor shroud can have an enormously beneficial effect on efficiency in micro-compressors. Now, even for a rather extreme compressor inlet temperature of $315 \mathrm{~K}$, the compressor outlet temperature is under $360 \mathrm{~K}$, where the highstrength titanium beta alloys can be used with excellent long-term performance.

The compressor bearing gas requirement is doubled from $\sim 0.25 \mathrm{~g} / \mathrm{s}$ to $\sim 0.5 \mathrm{~g} / \mathrm{s}$, but the total bearing gas load on the compressor is essentially unchanged, as the reduced expander bearing śize (from $4 \mathrm{~mm}$ to $2 \mathrm{~mm}$ ) reduces the total bearing gas flow in the two expanders from $\sim 0.6 \mathrm{~g} / \mathrm{s}$ to $\sim 0.35 \mathrm{~g} / \mathrm{s}$.

3.1 The Microturbine Expanders. The initial rotor design was approximately as shown in Figure 5 with turbine diameters of $7 \mathrm{~mm}$. The Nd-B-Fe generator core is epoxied into the center of the silicon-nitride bearing sleeve. Silicon-nitride rings, epoxied inside the bearing sleeve on either side of the magnet, capture the threaded titanium nuts that are used to secure the turbines to each end of the bearing. The fastening method was selected to minimize thermal stress and allow concentricity to be maintained within about $0.5 \mu \mathrm{m}$. Tests have shown the method to be reliable and unaffected by rapid thermal cycling down to $4.2 \mathrm{~K}$.

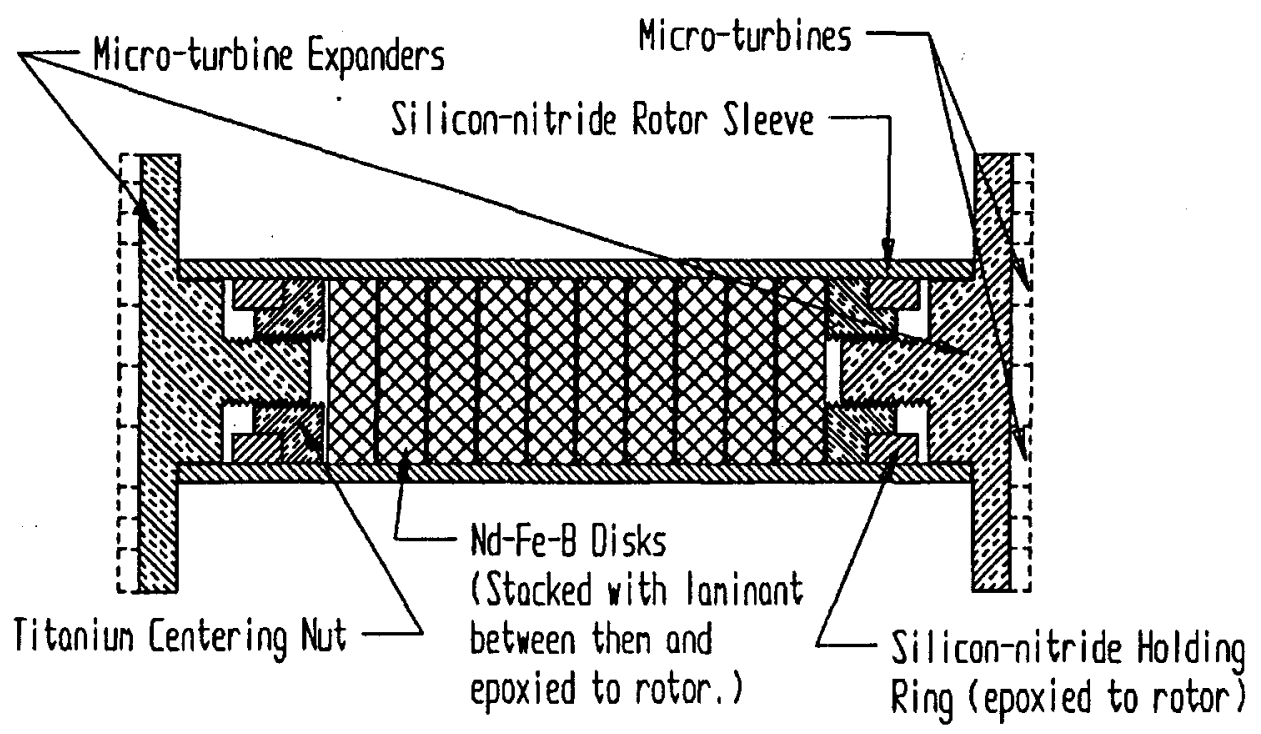

Figure 5. Initial, laminated, dipolar, permanent-magnet, cylindrical (PMC) generator core inside a silicon-nitride bearing sleeve with titanium radial-inflow turbines on each end. 
To date, six generations of expander assemblies have been designed and procured, and five have been manufactured and tested. The turbine caps and some of the stator components other than the bearing journal were machined from PEEK composites to save cost in initial experiments, though ultimately the entire stator will be silicon nitride. To expedite initial experiments, the first three versions used a $5 \mathrm{~mm}$ bearing diameter, as we had appropriate raw materials in stock. The third-generation expander utilized turbines from titanium alloy Beta-C to permit higher speeds (for better efficiency during the cool-down) and to better control tip clearance and leakage (CFD analysis later showed thermal conduction through the titanium blades to constitute a significant loss in polytropic efficiency. So subsequent designs reverted to composites.)

The first-generation expander had several problems including very high initial frictional losses. Several changes were made to the tapers, clearances, and manifolding which successfully reduced the frictional drag to at least a reasonable level - though still very high. We then ran into axial bearing stability problems that we determined required more extensive changes.

In Figure 6a (page 20), the second generation experimental expander has all the critical areas, components, and clearances labeled while the next has only the key differences pointed out. About half of the gas for the radial bearings exhausts into the annular space between the turbine blisks and bearing stator at each end to form the required axial thrust bearings [Doty 17], while the rest exhausts through holes in the central region of the bearing stator between the generator windings [Doty 11]. The first generation's lack of the "Axial Bearing Exhaust" path (shown in figure 6a) was determined to be the likely main contributor to the initial axial stability problems. Several improvements were made (as discussed in some detail in earlier reports) to improve bearing stability, reduce axial bearing friction, and attempt to reduce leakage between the drive plenum and the axial bearing exhaust.

Stability of the second-generation $5 \mathrm{~mm}$ experimental prototype was improved considerably (with bearing optimized for very fast spinning with a light load in helium at $\sim 120 \mathrm{~K}$ ), and bearing friction was reduced, though still somewhat high. Stable spinning was readily achieved for a wide range of bearing pressures (2-4 atm) at all speeds úp to $17 \mathrm{kHz}$ (the forces developed here are equivalent to $21 \mathrm{kHz}$ for a $4 \mathrm{~mm}$ bearing) and at temperatures down to $90 \mathrm{~K}$ with nitrogen.

To expedite generator experiments, the second-generation expander used metal (rather than ceramic) nozzle caps, and plastic turbine caps were used, which limited precision and hence sealing between the axial bearing and the turbine inlet plenum. Turbine efficiency was degraded by this leakage, as well as the increased leakage associated with imperfect fit between plastic manifold rings and ceramic and metal stator parts after thermal cycling. Also, there is substantial bearing friction at room temperature (RT), and about $25 \%$ of this heat would be conducted through the turbine blisks and metal nozzle caps, appearing directly as degraded turbine efficiency and making it difficult to separate these two factors. Neglecting all these effects, second-generation expander efficiencies at RT up to $26 \%$ were measured. 
Measurements of turbine exhaust flow and supply flow confirmed that the turbine inlet plenum leakage into the axial bearing exhaust was huge in many cases - possibly $25 \%$ of turbine inlet. Changes were made to the third-generation expander to tighten up these clearances and add a micro-labyrinth seal to the turbine inlet plenum.

Correcting for plenum leakages plus $\sim 0.3 \mathrm{~W}$ heat exchange from the bearing exhaust (total bearing losses 1.3-1.8 W at $6 \mathrm{kHz}$ ), plus $\sim 0.3 \mathrm{~W}$ stator core hysteresis loss suggests turbine efficiency may have been greater than $30 \%$ with air at $300 \mathrm{~K}, 4.9 \mathrm{~W}$ shaft power, $2.9 \mathrm{~W}$ electrical output power, at $5.8 \mathrm{kHz}$ in this expander for a pressure ratio of 2.0 and mass flow $\sim 0.2 \mathrm{~g} / \mathrm{s}$ for each turbine. Bearing friction was not much above expectations for room temperature. Results from several second-generation expander experiments and various conditions were shown in Table 1 below.

In Table $1, p_{\mathrm{T}}$ is the turbine inlet pressure, $G_{\mathrm{T}}$ is the total turbine mass flow, $p_{\mathrm{B}}$ is the bearing inlet pressure, $G_{\mathrm{B}}$ is the bearing mass flow, $f$ is the rotational frequency, $T_{1}$ is the turbine inlet temperature, $T_{2}$ is the turbine outlet temperature, $\eta_{T}$ is the turbine efficiency calculated from temperature drop, $R_{\mathrm{L}}$ is the generator load resistance (each winding pair), $V_{\mathrm{PP}}$ is the peak-to-peak voltage across the loads, $P_{\mathrm{O}}$ is the generator output power, $P_{\mathrm{B}}$ is the total bearing power loss (friction plus leakage momentum), $P_{\mathrm{O}}$ is the field core hysteresis loss, $P_{\mathrm{GL}}$ is additional generator losses (eddy currents in metal nozzle caps $+I^{2} R$ in field windings + rotor core eddies), $P_{\mathrm{S}}$ is shaft power $\left(P_{\mathrm{O}}+P_{\mathrm{B}}+P_{\mathrm{C}}+P_{\mathrm{GL}}\right), P_{\mathrm{T}}$ is maximum theoretical power available for $p_{\mathrm{T}}$ and $G_{\mathrm{T}}$, and $\eta_{\mathrm{E}}=P_{\mathrm{S}} / P_{\mathrm{T}}$.

Table 1. Second-generation Micro-expander Experiments

\begin{tabular}{|c|c|c|c|c|c|c|c|c|c|c|c|}
\hline & $\begin{array}{c}\text { Expr. \# } \\
\text { Units }\end{array}$ & 1 & 2 & 3 & 4 & 5 & 6 & 7 & 8 & 9 & 10 \\
\hline Gas & - & Air & Air & Air & Air & Air & Air & He & He & He & He \\
\hline$p_{\mathrm{T}}$ & $\mathrm{kPa}$ & 200 & 200 & 250 & 300 & 300 & 350 & 200 & 250 & 300 & 200 \\
\hline$G_{\mathrm{T}}$ & $\mathrm{g} / \mathrm{s}$ & 0.4 & 0.4 & 0.5 & 0.61 & 0.62 & 0.77 & 0.174 & 0.21 & 0.25 & 0.16 \\
\hline$p_{\mathrm{B}}$ & $\mathrm{kPa}$ & 195 & 195 & 202 & 222 & 263 & 243 & 304 & 345 & 362 & 209 \\
\hline$G_{\mathrm{B}}$ & $\mathrm{g} / \mathrm{s}$ & 0.14 & 0.15 & 0.17 & 0.210 & 0.25 & 0.29 & 0.13 & 0.16 & 0.19 & 0.38 \\
\hline$f$ & $\mathrm{~Hz}$ & 4700 & 5800 & 6400 & 7900 & 9400 & 9000 & 10100 & 14600 & 15300 & 9000 \\
\hline$T_{1}$ & $\mathrm{~K}$ & 297.1 & 297.1 & 297.1 & 297.1 & 297.1 & 297.1 & 297 & 297 & 297 & 140 \\
\hline$T_{2}$ & $\mathrm{~K}$ & 285.8 & 287.7 & 280.5 & 276.1 & 280.2 & 274.5 & 286.6 & 280.9 & 276.2 & \\
\hline$\eta_{\mathrm{T}}$ & $\%$ & 21.2 & 17.7 & 24.2 & 26.2 & 21.1 & 25.3 & 14.4 & 17.7 & 19.7 & \\
\hline$R_{\mathrm{L}}$ & $\Omega$ & 55 & 100 & 55 & 55 & 100 & 55 & 200 & 200 & 100 & 200 \\
\hline$V_{\mathrm{PP}}$ & $\mathrm{V}$ & 25 & 34 & 35 & 38 & 53 & 50 & 51.5 & 74.5 & 81 & 46 \\
\hline$P_{\mathrm{O}}$ & $\mathrm{W}$ & 2.84 & 2.89 & 5.57 & 6.56 & 7.02 & 11.40 & 3.32 & 6.94 & 16.40 & 2.65 \\
\hline$P_{\mathrm{B}}$ & $\mathrm{W}$ & 1.1 & 1.73 & 2.14 & 3.36 & 4.88 & 4.44 & 5.69 & 12.6 & 13.9 & 2.83 \\
\hline
\end{tabular}




\begin{tabular}{|c|c|c|c|c|c|c|c|c|c|c|c|}
\hline$P_{\mathrm{C}}$ & $\mathrm{W}$ & 0.24 & 0.29 & 0.32 & 0.4 & 0.47 & 0.45 & 0.51 & 0.73 & 0.77 & 0.45 \\
\hline$P_{\mathrm{GL}}$ & $\mathrm{W}$ & 0.06 & 0.03 & 0.12 & 0.14 & 0.08 & 0.25 & 0.02 & 0.04 & 0.2 & 0.004 \\
\hline$P_{\mathrm{S}}$ & $\mathrm{W}$ & 4.24 & 4.94 & 8.14 & 10.5 & 12.5 & 16.5 & 9.53 & 20.3 & 31.3 & 5.93 \\
\hline$P_{\mathrm{T}}$ & $\mathrm{W}$ & 21.4 & 21.4 & 34.2 & 48.5 & 49.5 & 68.6 & 65. & 100 & 137 & 28 \\
\hline$\eta_{\mathrm{E}}$ & $\%$ & $\mathbf{2 0}$ & $\mathbf{2 3}$ & $\mathbf{2 4}$ & $\mathbf{2 2}$ & $\mathbf{2 5}$ & $\mathbf{2 4}$ & $\mathbf{1 5}$ & $\mathbf{2 0}$ & $\mathbf{2 3}$ & $\mathbf{2 1}$ \\
\hline
\end{tabular}

Figures 6a, 6b, and 6c: Experimental Micro-turbine-expanders, (all clearances in mils, magnetic cores not shown in first and last expander).

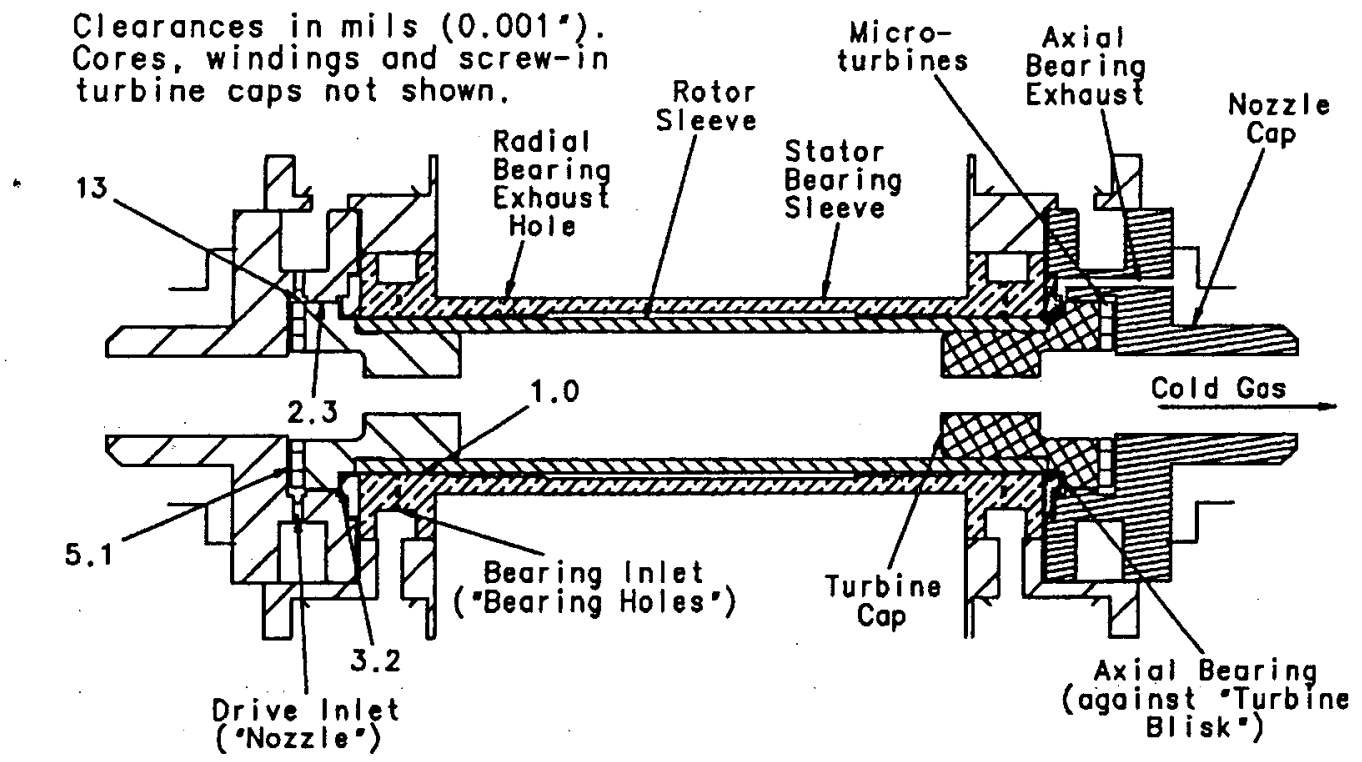

Figure $6 \mathrm{a}$, Second-generation: $5 \mathrm{~mm}$ bearing, $6 \mathrm{~mm}$ turbine OD.

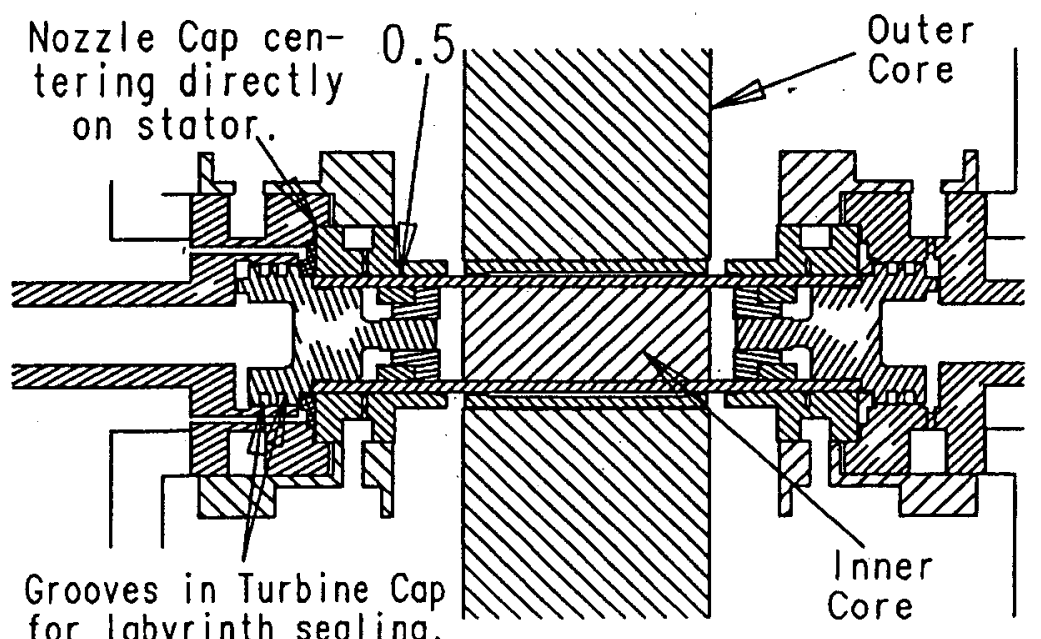

Fiqure $6 \mathrm{~b}$. Third-qeneration: $5 \mathrm{~mm}$ bearing. $6 \mathrm{~mm}$ turbine $\mathrm{OD}$. labvrinth seal. 
Fourth- and Fifith-generations were essentially a scaled-down version of above: $4 \mathrm{~mm}$ bearing, 5 $\mathrm{mm}$ turbine $\mathrm{OD}$, labyrinth seal.

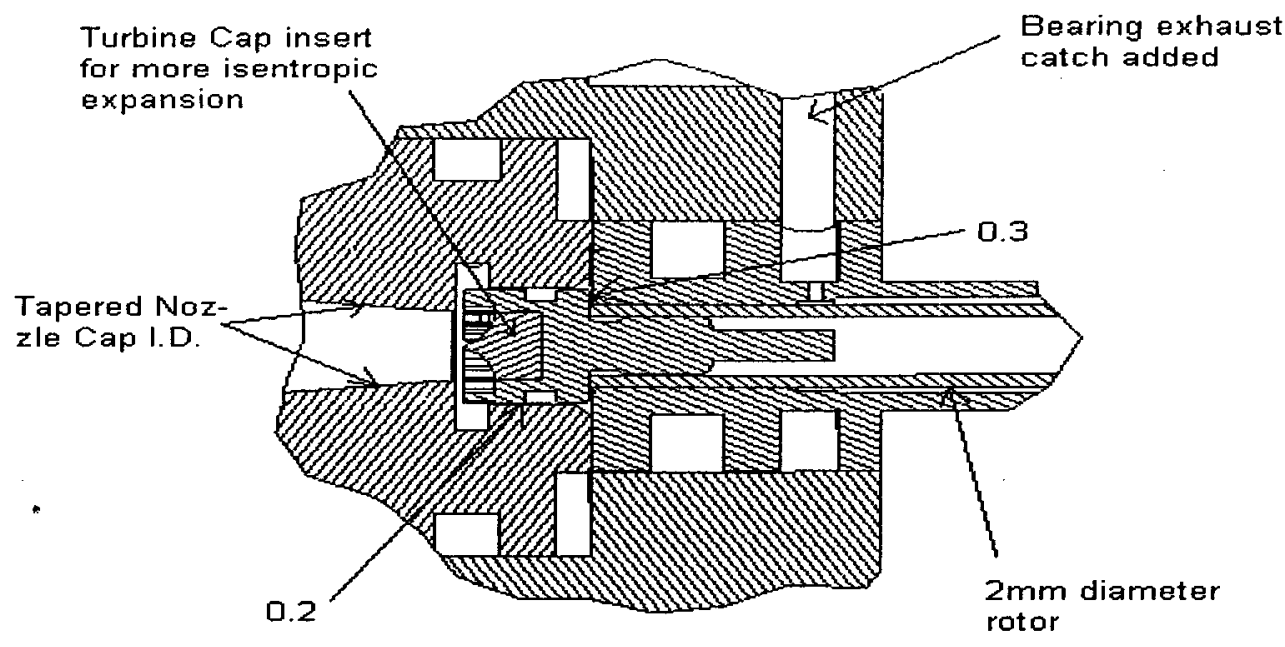

Figure $6 \mathrm{c}$, Sixth-generation: $2 \mathrm{~mm}$ bearing, $3 \mathrm{~mm}$ turbine $\mathrm{OD}$, other features as noted. follows:

The maximum theoretical turbine power $P_{\mathrm{T}}$ available for a perfect gas is calculated as

$$
P_{T}=G_{T} C_{P} T_{H}\left(1-1 / T_{M}\right)
$$

where $T_{M}$ is the maximum (ideal) temperature ratio:

$$
T_{M}=r^{((\gamma-1) / \gamma)}
$$

Using two methods of calculating efficiency $\left(\eta_{\mathrm{E}}\right.$ and $\left.\eta_{\mathrm{T}}\right)$ helps to quantify the various loss mechanisms. In Table 1, the largest uncertainties lie in the bearing friction losses. We should also note that in one case the expander (with a $5 \mathrm{~mm}$ bearing) was allowed (by mistake) to spin over $17 \mathrm{kHz}$, which was $\sim 20 \%$ beyond what our experience indicates should be the safe upper limit (due to the loading of the Nd-B-Fe rotor core on the silicon-nitride bearing sleeve) but no failure occurred.

Maximum vibrational displacement occurred at the highest spinning rates and was measured using a calibrated magnetic phonograph cartridge $(0.1 \mathrm{~V} /(\mathrm{m} / \mathrm{s}))$ with a diamond stylus by the method described in the Phase I Final Report. Maximum measured displacement (on this $0.02 \mathrm{~kg}$ expander) was about $0.1 \mu \mathrm{m}$ at $9 \mathrm{kHz}$ and about $0.05 \mu \mathrm{m}$ at $3 \mathrm{kHz}$ - which is at least an order of magnitude below application requirements, and the third-generation expander's vibration amplitude (though not measured) is comparable or lower as the radial clearances are less.

Changes in the third-generation optimization (see Figure $6 \mathrm{~b}$ ) reduced bearing leakage from the following changes: lengthening the cylindrical bearing regions (from $3.1 \mathrm{~mm}$ in the 
second expander's bearing to $6 \mathrm{~mm}$ in the third expander's bearing), reducing bearing clearance about $50 \%$, reducing the bearing orifices about $30 \%$, adding length and intricacy to the microlabyrinth turbine-cap seal, and reducing the turbine-cap seal gap (this was possible due to the use of titanium micro-turbines).

Such large changes in several key parameters simultaneously is always risky, and the affects on the radial bearing stability were not entirely what we had hoped for. Although the bearing friction was surprising low (with such tight, long clearances), the necessary bearing pressure increased. The high bearing pressure was necessary down to the lowest temperature tested $(115 \mathrm{~K})$ with $\mathrm{He}$; and, at this temperature, it was joined by an unacceptably high bearing flow rate. We believe that this is from an over-correction and that an optimum can be achieved by increasing the bearing clearance in the next version.

Some of the other changes incorporated into the third-generation expander did yield the expected benefits. As a result of the turbine-cap changes (titanium, small-gap, O.D. grooves), the sealing between the drive and bearing flows was excellent, even up to $\sim 6 \mathrm{~atm}$. of bearing pressure. The high bearing pressure required for even a few $\mathrm{kHz}$ spinning speed made it impossible to fully assess the turbine performance, but the turbines/nozzles appeared to perform well from RT down to $115 \mathrm{~K}$, where the maximum speed attained was only $1.7 \mathrm{kHz}$ - due to the bearing problem.

Compromise changes in the bearing parameters were introduced in the 4th generation, 6 $\mathrm{mm}$ expander with a $4 \mathrm{~mm}$ bearing, which was recently completed and preliminary experiments were performed. Very recently, we have determined that some of the axial stability problems seen in our $4 \mathrm{~mm}$ NMR sample spinners arise from acoustic resonances in chambers that are in or adjacent to the exhaust stream. Simple and effective methods of suppressing these resonances have been developed and are expected to also prove beneficial in improving bearing stability of the cryo-expander.

Data from the fourth generation expander testing showed inconsistency, with much lower efficiency from the turbine at one end than at the other end. Inspection revealed that this was due to excessive leakage from machining errors at both ends, but more pronounced at one end. However, it was obvious that the reduction from $5 \mathrm{~mm}$ to $4 \mathrm{~mm}$ was not sufficient to achieve the necessary magnitude of reduction in frictional losses and increase in expander efficiency for a usable cryocooler in the 1-2 $\mathrm{W}$ range.

Preliminary calculations indicated that scaling the turbine diameter down by a factor of two should permit operation at full inlet admission with greatly improved turbine efficiency. This was, of course, recognized from the beginning, but it was expected that manufacturing problems, especially for the $2 \mathrm{~mm}$ silicon-nitride rotor sleeve and gas-bearing stator, would make such a small turbine prohibitively difficult to manufacture. At this point, we had no choice but to proceed and find solutions to the micro-manufacturing challenges that are sure to arise. This redesign is complete, and we were able to find the necessary diamond hones. We procured silicon-nitride parts HIP'd to near net shape for the smaller expander bearing sleeve from an alternate source, as our prior source has declined to quote on this part. The preliminary part of the stator and rotor 
work has been completed to help establish feasibility of the new approach.

\subsection{Computational Fluid Dynamics (CFD).}

3.2.1 CFD Software Selection. While CFX TASCflow clearly established the leadership position in the field of CFD for turbomachinery over the past decade, Numeca (recently formed out of the advanced CFD programs from three departments at the University of Brussels) seems to offer a number of distinct advantages for improved CFD in turbomachinery. After a careful evaluation of these two leading commercial options for CFD turbo-machinery optimization, we concluded that the Numeca's FINE/Turbo was clearly the best option for our applications.

There appear to be several fundamental advantages to the approach taken in their solver. First of all, their use of second-order density-based pre-conditioned Navier Stokes equations allows cells with aspect ratios (maximum cell dimension to minimum cell dimension) greater than 2000 without loss in accuracy. This is an order of magnitude higher aspect ratio than is recommended in the competing codes. As a result, the mesh may be structured to have much smaller dimensions in the direction of velocity gradients where the gradients are high without a prohibitive increase in the number of cells. Secondly, the mesh is structured so as to automatically perform the desired number of iterations first on a course grid, then on a medium grid, and finally on a fine grid. As a result, problems will typically converge much faster for the same level of accuracy, and the time required to get a fairly accurate indication of whether or not the solution will converge is often only a few percent of the time needed by competing codes.

Thirdly, the Numeca code can easily be configured to use any of three standard mathematical models (laminar Navier Stokes, Euler, or turbulent Navier Stokes) with any of seven turbulence models (Baldwin-Lomax, Chien $k-\varepsilon$, standard $k-\varepsilon$, Launder-Sharma $k-\varepsilon$, Yang-Shih $k-\varepsilon$, non-linear high-Re $k-\varepsilon$, and non-linear low-Re $k-\varepsilon$ ). The first two of these turbulence models (which our experiements showed to have advantages for most of our applications) are not available in CFX. We performed a series of careful experiments on micro-nozzles $(0.34 \mathrm{~mm}$ diameter) and found the Numeca results to agree with our experiments within $2 \%$ over a wide range of conditions (Mach numbers from 0.5 to 1.5), while TASCFlow errors were usually a little larger, (and it was easy to set up the run with improper time steps and see much larger errors). We also found it much easier to impose the desired thermal conditions at the walls in FINE/Turbo.

However, we felt that the most significant advantages of Numeca were (1) the effectiveness of it's automatic grid generator and (2) it's vastly simplified file management system. While there is undeniably a longer learning curve with Numeca's Autogrid than with CFX's Turbogrid, the methods used in the Numcea code to determine the cell clustering and structuring make it much easier (after the learning curve) to consistently obtain high-quality grids with much less effort as changes are made in the geometry. Finally, CFX's antiquated and patch-meal data management system has grown over the past two decades into a hodge-podge of 135 obscure files in 14 subdirectories for each computation. In contrast, the Numeca code typically uses a total of seven compact, intelligible files in standard data formats. 
3.2.2 Simulations on Microturbine Expanders. For the initial $6 \mathrm{~mm}$ turbine expander, we began with a simple blade design similar to what we have used successfully in our $10 \mathrm{~mm}$ NMR MAS products (which have a $7 \mathrm{~mm}$ turbine). We began by scaling to $6 \mathrm{~mm}$, increasing the number of blades to 16 , and optimizing leading and trailing edge angles for best efficiency at tip surface speeds about half the speed of sound, or $\sim 8 \mathrm{kHz}$ for the $6 \mathrm{~mm}$ turbine with $\mathrm{N}_{2}$ at RT. This is well below the optimum speed when the turbine is evaluated alone, but probably close to optimum when bearing losses are considered. Recall that the bearing losses are quadratic with rotational rate while the efficiency is a slowing varying function of frequency, mildly peaked at maximum tip speeds $\sim 70 \%$ of the speed of sound.

One of the most important revelations from the early simulations was the effect of heat transfer at the walls on micro-turbine efficiency. At conventional turbine sizes and even at more common micro-turbine sizes (several orders of magnitude larger flow rate than our case), the effect of heat transfer at the walls is of interest only when one is attempting to cool the blades for improved blade strength - the heat transfer has no effect on aerodynamic efficiency. Hence, it is common for uncooled blades to be simulated with the assumption of adiabatic walls. We suspected that heat conduction through the metallic (titanium) blade may be a factor in the reduced experimental efficiency of the titanium blade compared to the plastic composite blade and decided to simulate it. The simulations showed the effects to be even larger than expected, especially for the initial, short-span blades with helium at low temperatures. For nitrogen at 130 $\mathrm{K}, 260 \mathrm{kPa}$ inlet, $0.55 \mathrm{~g} / \mathrm{s}$, the efficiency decreased by $\sim 10 \%$ when going from adiabatic blade walls to isothermal blade walls at the mean of the inlet and outlet total temperatures. The relative loss in efficiency was about the same for the $3 \mathrm{~mm}$ rotor with $0.3 \mathrm{~g} / \mathrm{s}$ of helium at $50 \mathrm{~K}, 230 \mathrm{kPa}$ inlet.

Even though the thermal conductivity of titanium $(\sim 7 \mathrm{~W} / \mathrm{m} / \mathrm{K})$ is more than an order of magnitude lower than that of copper $(400 \mathrm{~W} / \mathrm{m} / \mathrm{K})$ at room temperature, it is still high enough for the walls to be nearly isothermal. Clearly, there is the potential for substantial efficiency advantage from using turbines of plastic composite rather than metal at this size, as the thermal conductivity of the glass-filled composites at low temperature $(-0.1 \mathrm{~W} / \mathrm{m} / \mathrm{K})$ is nearly two orders of magnitude lower than titanium's and low enough for the wall conditions to be essentially adiabatic. However, it is not yet clear whether or not we can obtain adequate long-term reliability under repeated thermal cycling with composite turbines.

We have continued to use tapered, round (drilled) nozzles to simplify manufacturing. Improvement in efficiency should be possible from more optimally contoured inlet guiding vanes, similar to those illustrated in Figure 7. However,

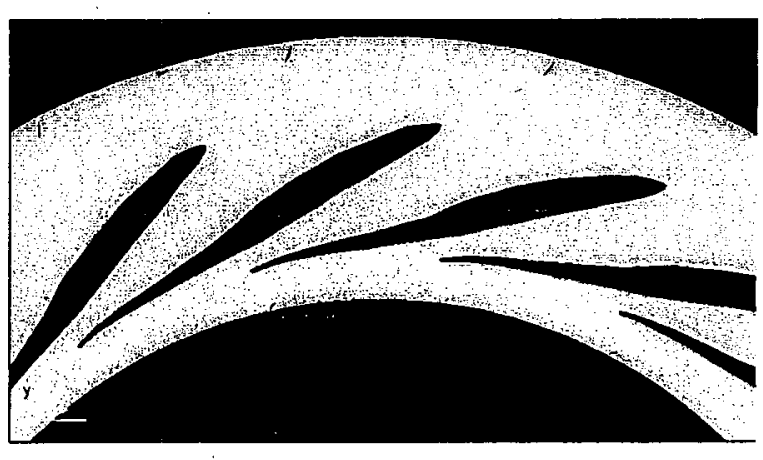

Figure 7. Inlet guiding vanes. for the present, the round-hole nozzles are sufficient. With a modest amount of effort on the blade profile optimization, we were able to get simulated polytropic efficiencies above $46 \%$ at 8 $\mathrm{kHz}(480,000 \mathrm{rpm})$ with air at room temperature over the range of 205 to $275 \mathrm{kPa}$ for round-hole 
nozzles.

Of course, the gross efficiencies ignore bearing losses, which as we have noted, have cut effective net efficiencies by about a factor of two thus far. We will shortly show that these losses can be effectively addressed for the cryo expanders, but first we present some conclusions from experiments and simulations of the current $6 \mathrm{~mm}$ expanders at room temperature.

As previously noted, most of the prototype parts (other than the bearing sleeve and stator) have been made of composites (glass-filled PEEK) rather than silicon nitride to reduce prototyping expenses. As a result it has been very difficult to limit leakages (a) from the nozzle outlet plenum past the labyrinth seal into the axial bearing exhaust (see Figure 6a) and (b) sometimes even from the nozzle inlet plenum into the axial bearing exhaust. Also, it has been difficult to adequately control the tip clearance - partly because the axial centering of the rotor depends on minute details in the way the turbine mounts against the bearing sleeve, as this effects the axial bearing flow.

With a large leakage from the nozzle exhaust plenum, the rotor mass flow is well below where it should be, so the rotor efficiency, as determined from the outlet total temperature, would be expected to be below the simulated efficiency. But there is another problem in comparing the rotor efficiency at room temperature determined from the measured outlet temperature with the initial simulated results. The bearing gas, heated by friction, flows over the back side of the hub,

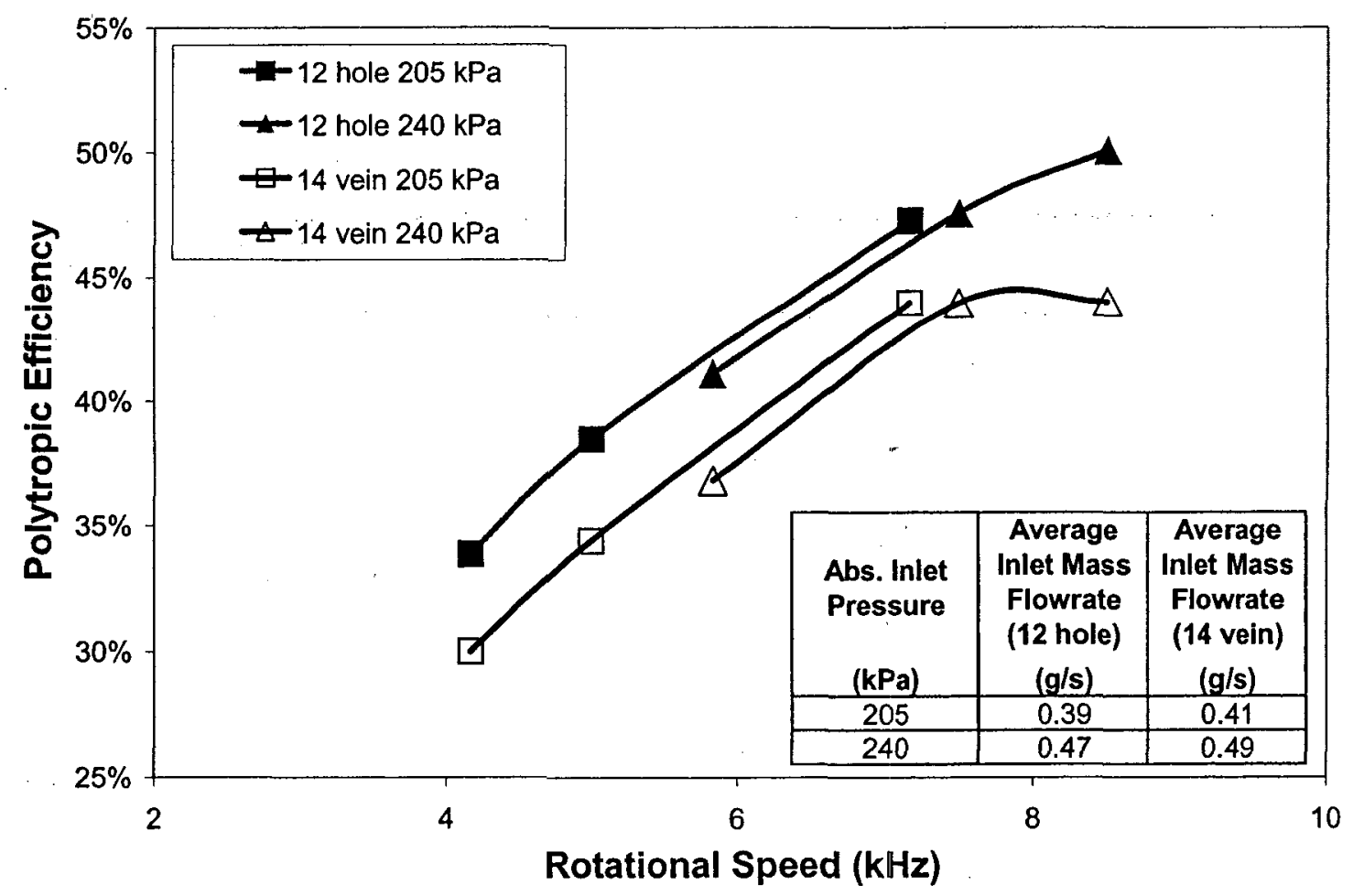

Figure 8. Comparison of stage simulations run with a 16 blade expander (6 $\mathrm{mm} \mathrm{dia)} \mathrm{and} \mathrm{two}$ different nozzle designs: (1) 12 hole $(0.34 \mathrm{~mm} \mathrm{dia})$ nozzle and (2) 14 vein nozzle at $296 \mathrm{~K}$. 
and a portion of this heat is conducted into the rotor stream through the hub and blades. For a 4 $\mathrm{mm}$ rotor with a nitrogen gas bearing at room temperature spinning at $8 \mathrm{kHz}$, the end-bearing exhaust is heated $\sim 16^{\circ} \mathrm{C}$ above the inlet temperature while the mean of the inlet and outlet rotor temperatures should be $\sim 24^{\circ} \mathrm{C}$ below the inlet temperature. The mean surface temperature for a titanium hub should then be $\sim 10^{\circ} \mathrm{C}$ below the inlet total temperature. Repeating the simulations with isothermal blade/hub walls at this temperature is expected to show another $10 \%$ drop in efficiency $(\sim 1.2 \mathrm{~W}$ is conducted through the titanium hub for an axial temperature gradient of $25^{\circ} \mathrm{C}$ ), which will make simulated efficiency close to the experimental results. With a composite hub, this effect is reduced by a factor of $\sim 20$ at room temperature and $\sim 70$ at low temperatures, but it becomes much more difficult to control tip clearance and plenum leakage.

Figure 8 shows some simulation results for the $6 \mathrm{~mm}$ turbine with nitrogen at room temperature. Some of the characteristics and assumptions include adiabatic (composite) walls, $0.03 \mathrm{~mm}$ tip gap, 16 blades, $0.35 \mathrm{~mm}$ blade span at the leading edge, and $\sim 0.45 \mathrm{~mm}$ span at the trailing edge. In all cases, the simulations were run for the complete stage with imposed inlet total pressure and total temperature and outlet static pressure. (The indicated mass flows are for a single turbine.) The rotor mesh size was about $70 \mathrm{~K}$ points, with minimum cell widths of 0.0015 $\mathrm{mm}$ (corresponding to $\sim 3 \mathrm{y}+$ ). The nozzle mesh size was $\sim 30 \mathrm{~K}$ points for the inlet guiding vanes, but $\sim 150 \mathrm{~K}$ cells were needed here for the round-hole nozzles. Both the rotor and nozzle used Imeshes with Numeca's standard geometric clustering algorithms transverse to the flow direction, but with aspect ratios increased by about a factor of 5 in the flow direction at the leading and trailing edges. Efficiencies are shown both with the round-hole nozzles (12 converging nozzles, $0.34 \mathrm{~mm}$ throat diameter), and with 14 inlet guiding vanes similar to those shown in Figure 7 , where the minimum throat passage width is $-0.21 \mathrm{~mm}$.

A $3 D$ rendering of the simulated turbine is shown in Figure 9 with the mesh superimposed on the hub surface, and a close up of the blade is illustrated in Figure 10.

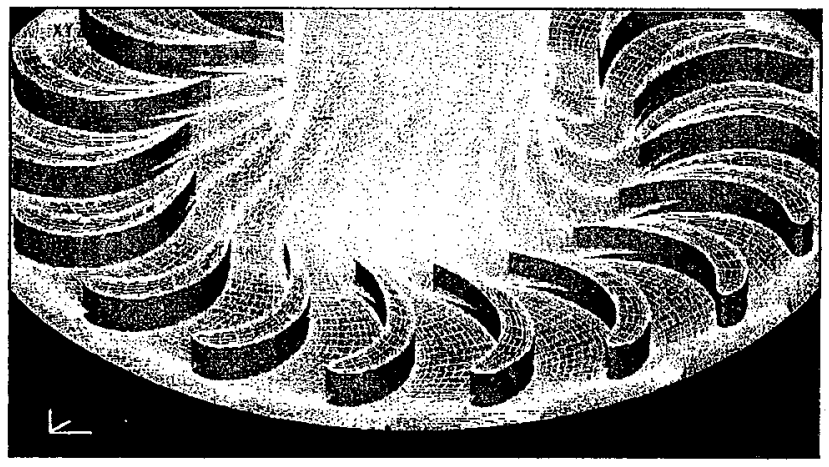

Figure 9. $6 \mathrm{~mm}$ expander turbine.

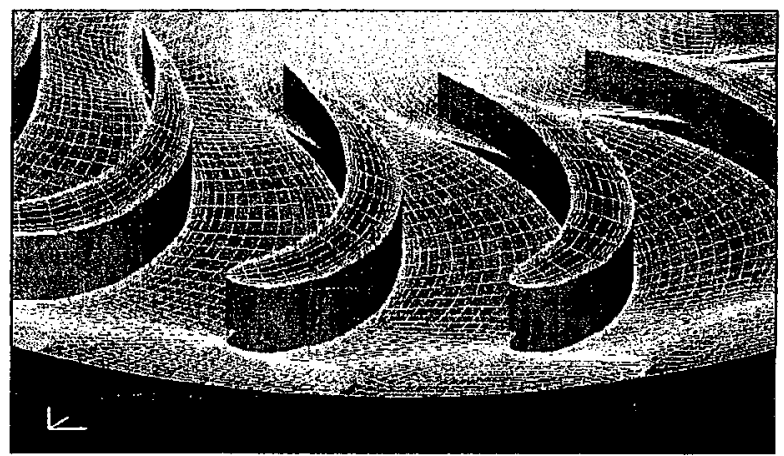

Figure 10. Close-up on the blade.

Some experimental data for the $6 \mathrm{~mm}$ expander with room temperature nitrogen and 12 converging round nozzles are presented in Figure 11. The efficiencies reported are the mean of the measurements (based on outlet temperature) for the two separate ends. The total inlet drive mass flow was also measured and was typically $\sim 20 \%$ larger than the sum of the outlet flows. 


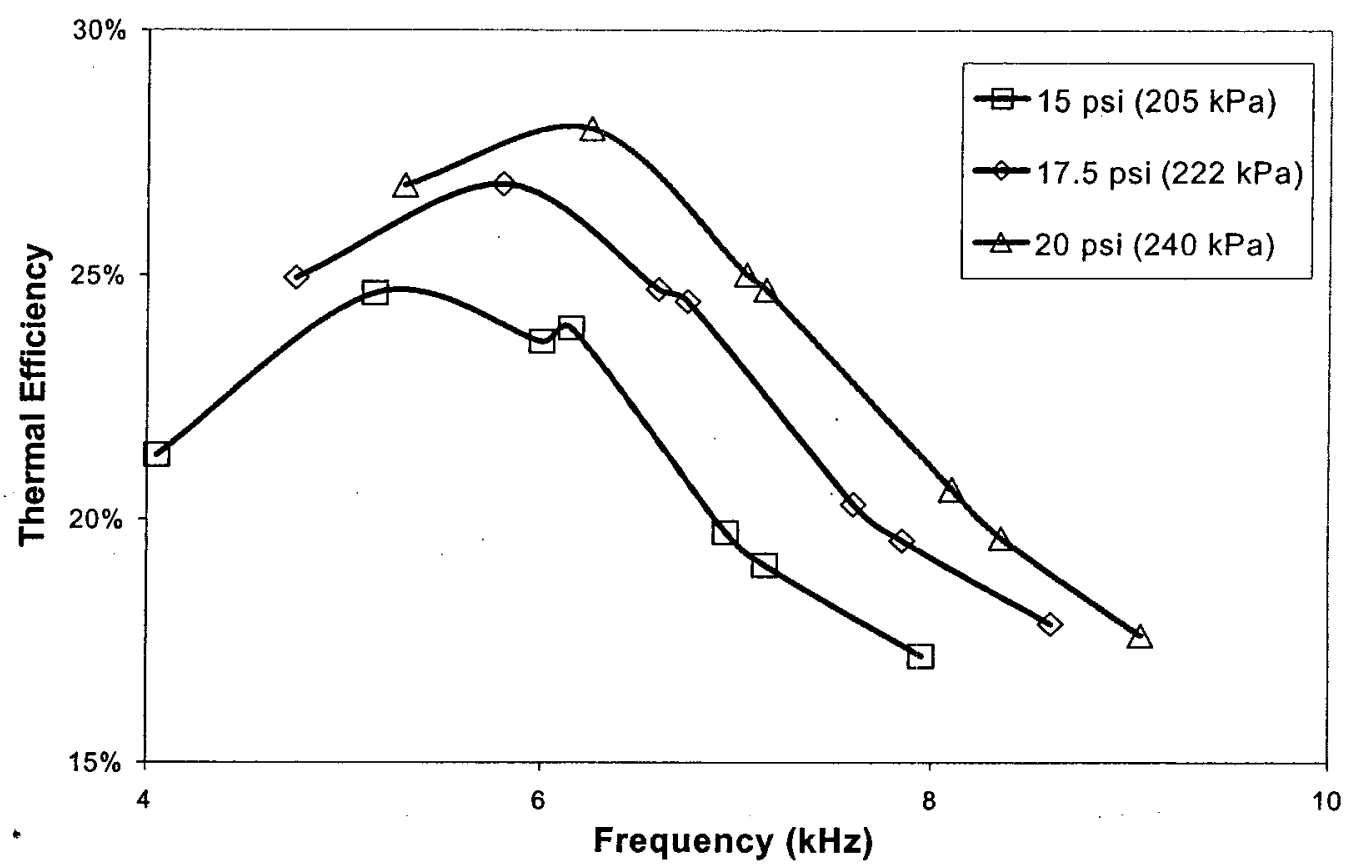

Figure 11. Thermal efficiency for a $6 \mathrm{~mm}$ expander and 12 hole $(0.34 \mathrm{~mm}$ dia) nozzle at $300 \mathrm{~K}$, both made of Glass-Filled PEEK, at three different inlet pressures.

We expect nearly a factor of three reduction in bearing friction as we scale down by a factor of two in diameter (bearing surface speed remains constant, surface area drops by about a factor of four, and clearance decreases by $\sim 25 \%$ ). Preliminary simulations on the $3 \mathrm{~mm}$ rotor show that its efficiency will be at least $10 \%$ higher than that of the $6 \mathrm{~mm}$ rotor because the span/diameter ratio is increased by nearly a factor of two to maintain the same mass flow. This improved turbine performance, along with the reduction in bearing friction and a drop in the bearing gas mass flow rate by nearly a factor of 2 should permit over $40 \%$ net efficiency in the next generation $3 \mathrm{~mm}$ cryo expanders at $0.3 \mathrm{~g} / \mathrm{s}$ per turbine.

With a $3 \mathrm{~mm}$ turbine diameter, it becomes impractical to have more than $\sim 13$ blades $(0.16$ $\mathrm{mm}$ cutter tool clearance) in a composite or $\sim 11$ blades $(0.22 \mathrm{~mm}$ cutter tool clearance) in titanium. So we began the simulations with 11 blades and restricted our evaluation to readily manufacturable designs - no twist, conical profiles at both the hub and the shroud, and no mixed flows.

Reducing the number of blades from 18 to 11 has a profound effect on optimum blade shape. A preliminary blade shape giving over $60 \%$ efficiency for $0.3 \mathrm{~g} / \mathrm{s}$ helium at $50 \mathrm{~K}$ is shown in Figure 12. To allow the rotor to spin safely at twice its normal operating rate during cool down, a large fillet (or chamfer) is included at the root of the blade for strengthening. The same blade shape with the span reduced approximately in half also gives good initial

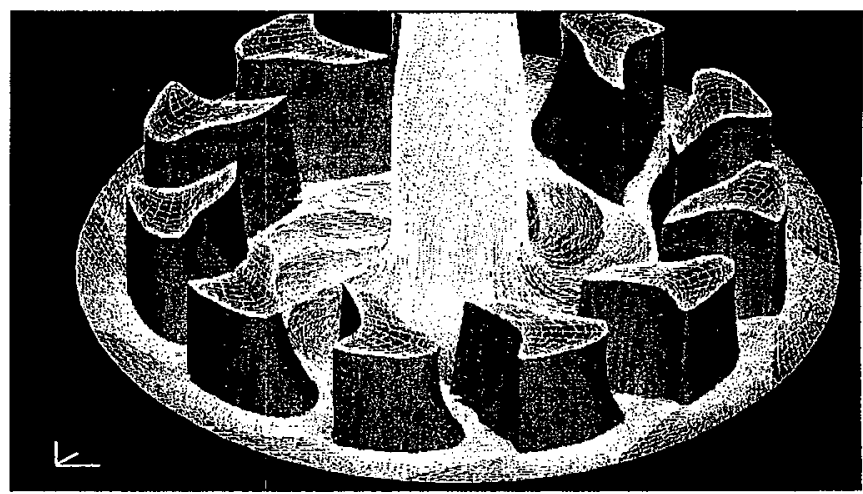

Figure 12. Preliminary 11 blade $3 \mathrm{~mm}$ rotor for $50 \mathrm{~K} .0 .3 \mathrm{a} / \mathrm{s}$ 
performance ( $\sim 50 \%$ efficiency) for $0.3 \mathrm{~g} / \mathrm{s}$ helium at $10 \mathrm{~K}$. Quite a bit of work remains to be done on the optimization of the $3 \mathrm{~mm}$ turbines both for $50 \mathrm{~K}$ and for $10 \mathrm{~K}$, but the software we wrote over the past six months for geometry manipulations on radial-flow turbine blades now makes this aspect of the project straightforward.

3.2.3 Simulations on the Compressor. The CFD simulations of the compressor showed us that the outlet temperatures will be higher than initially expected, and we learned that the long-term rupture strength of the titanium beta alloys drops sharply above $370 \mathrm{~K}$. Table 2 shows some data, including long-term rupture strength, for several materials at elevated temperatures. Note the factor-of-two drop in the 7-yr strength of Ti-beta-C as the temperature increases from $360 \mathrm{~K}$ to $410 \mathrm{~K}$. The maximum tip speed indicated assumes a design optimized for high speed.

\begin{tabular}{|c|c|c|c|c|c|c|c|c|c|}
\hline Material & $\begin{array}{c}\text { Density } \\
\text { d }\end{array}$ & $\begin{array}{l}\text { Therm. } \\
\text { cond., } \\
@ 400 \\
4\end{array}$ & $\begin{array}{c}\text { Rupture } \\
\text { Data } \\
\text { Temp. }\end{array}$ & $\begin{array}{c}0.1 \mathrm{hr} \\
\text { Yield } \\
\text { strength }\end{array}$ & \begin{tabular}{|c|}
$1000 \mathrm{hr}$ \\
Rupture \\
Strength \\
(RS)
\end{tabular} & \begin{tabular}{|c|}
$7 \mathrm{yr}$ \\
Rupture \\
Strength \\
$60,000 \mathrm{hr}$
\end{tabular} & $\begin{array}{l}0.1 \mathrm{hr} \\
\mathrm{RS} / \mathrm{d}\end{array}$ & $\begin{array}{l}7-y r \\
R S / d\end{array}$ & $\begin{array}{l}7-y r \\
v_{\text {tip }}\end{array}$ \\
\hline & $\mathrm{kg} / \mathrm{m}^{3}$ & $\mathrm{~W} / \mathrm{mK}$ & K & $\mathrm{MPa}$ & $\mathrm{MPa}$ & $\mathrm{MPa}$ & $\begin{array}{c}(\mathrm{m} / \mathrm{s})^{2} \\
\times 10^{3}\end{array}$ & $\begin{array}{l}(\mathrm{m} / \mathrm{s})^{2} \\
\times 10^{3}\end{array}$ & $\mathrm{~m} / \mathrm{s}$ \\
\hline A-JCN3030 & 1,450 & 0.5 & 370 & 120 & $\sim 90$ & $\sim 70$ & 83 & -48 & -370 \\
\hline Al-6061T6 & 2,700 & 180 & 340 & 268 & -160 & $\sim 95$ & 100 & $\sim 35$ & -320 \\
\hline Al-7075T6 & 2,800 & 130 & 340 & 480 & 420 & 250 & 178 & 90 & 510 \\
\hline Ti-Beta-C & 4,820 & 8 & 360 & 1300 & 1,100 & 800 & 270 & 166 & 700 \\
\hline Ti-Beta-C & 4,820 & 8 & 410 & 1,250 & 700 & 400 & 260 & 83 & 490 \\
\hline U-720 & 7,900 & 18 & 740 & 1200 & 1,100 & 1,000 & 152 & 126 & 600 \\
\hline $\mathrm{Si}_{3} \mathrm{~N}_{4}$ & 3,250 & 24 & 1200 & 500 & 500 & 500 & 154 & 154 & 640 \\
\hline
\end{tabular}

Even with $60 \%$ polytropic efficiency in each stage, with two compressors in series with an intercooler the outlet temperature of the second compressor could be up to $420 \mathrm{~K}$. The bottom line is that higher tip speeds are needed for a 2-stage design than can possibly be sustained by any available material under long-term operation. So we have decided to go with four centrifugal compressors (with intercool) so that balanced pairs can be used to simplify the axial gas-bearing design problem. Now, even for a rather extreme compressor inlet temperature of $320 \mathrm{~K}$, the compressor outlet temperature is under $370 \mathrm{~K}$ and the maximum blade temperature (for small titanium blades) is $<360 \mathrm{~K}$, where the high-strength titanium beta alloys can be used with excellent long-term performance. (For composite blades, the peak blade temperature is very close to the outlet temperature.)

The ideal cycle efficiency will be improved, as we now propose three intercoolers instead of one. Of course, the compressor bearing gas requirement is doubled from $\sim 0.25 \mathrm{~g} / \mathrm{s}$ to $\sim 0.5$ $\mathrm{g} / \mathrm{s}$, but the total bearing gas load on the compressor is unchanged, as the reduced expander 
bearing size (from $4 \mathrm{~mm}$ to $2 \mathrm{~mm}$ ) reduces the total bearing gas flow in the two expanders from $\sim 0.55 \mathrm{~g} / \mathrm{s}$ to $\sim 0.3 \mathrm{~g} / \mathrm{s}$.

Even with a 4-stage compressor, it appears that tip speed will need to be close to 600 $\mathrm{m} / \mathrm{s}$, and this severely limits the blade design options, as the blades must be nearly radial at the trailing edge. Of course, the hub must be tapered, and substantial fillets or chamfers are required at the root of the blade. Manufacturing a twisted mixed-flow blade from Ti-beta-C at these dimensions would be very difficult. However, it appears that most of the benefits of the twistedblade can be realized in a manufacturable way by combining a small axial-flow booster turbine on the same shaft immediately ahead of the centrifugal rotor. The axial booster turbine without stator vanes does not raise the static pressure at the inlet to the centrifugal compressor, but the total pressure and tangential velocity component are increased, which help the performance of the centrifugal stage.

A considerable amount of effort has already been put into the compressor design, but we have just figured out that there appears to an opportunity for a major improvement, so we are more or less back to an early stage of the optimization process. This break-through was the realization that heat transfer to the shroud for helium at this scale is extremely significant, so it should be possible to achieve a substantial improvement in efficiency by water-cooling the shroud to near room temperature $(\sim 312 \mathrm{~K})$. Indeed, the simulations show the apparent adiabatic efficiency (for a $0.09 \mathrm{~mm}$ tip gap) to increase from $\sim 70 \%$ to $\sim 90 \%$ when changing from adiabatic wall conditions to isothermal walls, where the shroud walls are at $312 \mathrm{~K}$, the blade walls at $342 \mathrm{~K}$, and the hub wall is at $338 \mathrm{~K}$. Of course, the compressor is no longer adiabatic, so the apparent adiabatic efficiency is meaningless. The more important parameter is the mechanical efficiency, given by the ratio of ideal shaft power to actual shaft power (the reciprocal of the definition for the expander turbine). For the adiabatic case, the mechanical efficiency and the shaft efficiency are, of course, the same $-70 \%$. When the shroud is water cooled, the mechancial efficiency is found to increase by $\sim 10 \%$ with the current blade design.

This magnitude of the improvement from water cooling of the shroud is quite surprising, as it is negligible in conventional compressor design. It's significance in our case comes from the conditions being far from conventional experience in three respects: (1) mass flow several orders of magnitude below that of typical small compressors, (2) low pressure ratio per stage $(\sim 1.3)$ and hence low mach numbers, and (3) relatively large tip gap ( $\sim 4 \%$ of the mean span). There may also be some increased significance from gamma being 1.67 rather than 1.4 , but the low molecular weight of our gas should have the opposite effect on the significance of isothermal shroud walls for a given mass flow and mach number.

To withstand tip speeds of $\sim 600 \mathrm{~m} / \mathrm{s}$, both the hub and the shroud must be sloped, which complicates manufacturing - especially at this scale, where tip leakage is critical. Also, the blades must be severely thickened near mid-chord at the root, and trailing-edge sweep-back is out of the question. In fact, even with large fillets at the root of the blades, they must re-curve so that the exit angle is within $\sim 25^{\circ}$ of radial to withstand the huge centrifugal forces. This costs several percent in efficiency but it is essential for blade strengthening. 
Figure 13 shows a $3 D$ rendering of the current 9-blade $22 \mathrm{~mm}$ design with a 2D mesh superimposed on the hub surface. (Rotation direction is clockwise is this view.) Quite a bit of detail work remains, and it is quite likely that the optimum number of blades would increase to 1013 and the optimum shape would change considerably now with the cooled shroud and isothermal blades.

The hub view of Figure 13 includes a portion of the extension surface that is the hub-

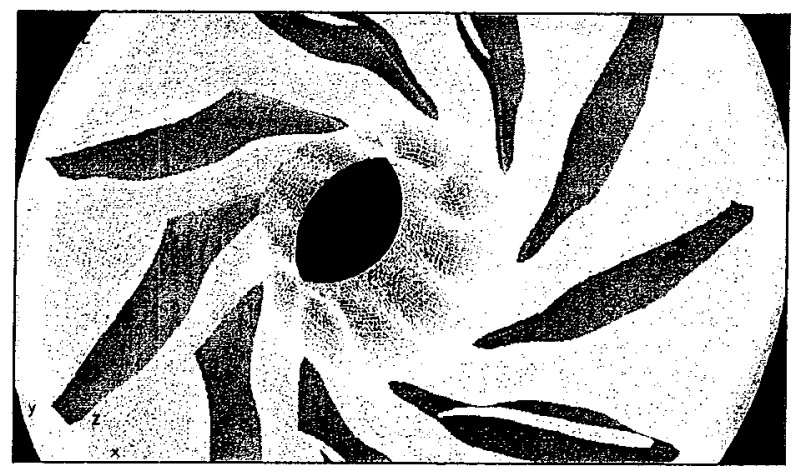

Figure 13. Preliminary 9-blade $22 \mathrm{~mm}$, $2.5 \mathrm{~g} / \mathrm{s}$ centrifugal helium compressor rotor. side of the stator outlet plenum. The actual rotor hub cannot extend beyond the maximum blade diameter for stress reasons, but it is easier to specify this surface as a non-rotating hub outlet extension surface than as an outlet stator section in Numeca's Autogrid.

With realistic values for the tip gap $(0.08-0.09 \mathrm{~mm})$, there have been difficulties in getting CFD convergence, so most of the CFD work thus far has assumed a tip gap of $0.05 \mathrm{~mm}$, which is probably unrealistic for long-term operation of a $22 \mathrm{~mm}$ turbine at $\sim 600 \mathrm{~m} / \mathrm{s}$ tip speed with gas bearings. Part of the reason for the difficulty in convergence comes from the small number of blades (dictated partially by both manufacturing and strength issues) and low mach numbers (maximum meridionally averaged mach number is less than 0.5), where standard turbulence models perform poorly. The low-speed preconditioning available in the Numeca turbulence code helps with this problem, but computation time is increased.

The meridional view of one stage of the centrifugal compressor is shown in Figure 14 with a $0.08 \mathrm{~mm}$ tip gap (barely discernably at the resolution in this figure) and meridionally averaged relative velocity vectors. At this point, it does not appear that stator diffuser vanes will be of benefit in the initial outlet region. The outlet plenum tapers down to about half the trailing-edge span at a radius of $\sim 16 \mathrm{~mm}$. As the gas spirals outward in this plenum, consevation of angular momentum

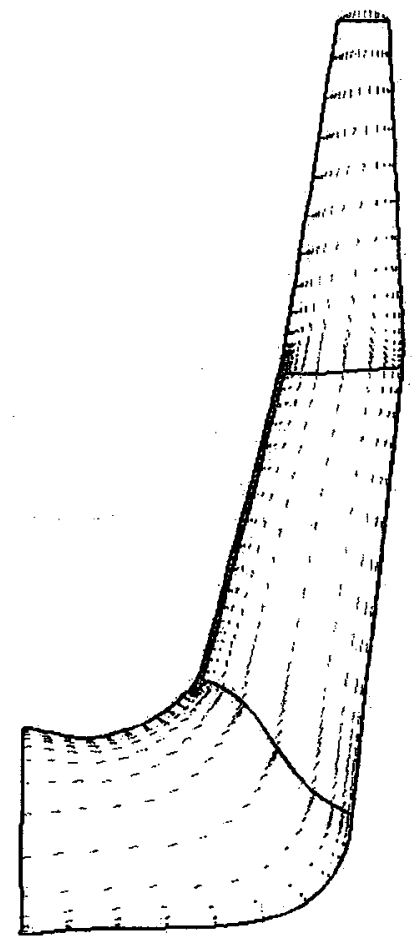

Figure 14. Meridional view of one stage of the centrifugal comressor. forces an efficient conversion of total pressure to static pressure. (The Numeca code is capable of capturing this process more accurately than alternative codes, which build artificial walls to stop backflow at the outlet.) Outlet guiding vanes and volute will be added beyond the vaneless radial diffuser section, but they are not expected to have much effect on efficiency. 
3.3 The Micro-generator. The problem of the micro-turbine generator was solved and sufficiently optimized during the Phase I for initial purposes. Hence, all that was done on this component during the first year of the Phase II was scaling the generator design down from the 5 $\mathrm{mm}$ rotor to the $4 \mathrm{~mm}$ rotor and making the appropriate new parts.

Fortunately, analysis in the last year shows that efficiency will still exceed $96 \%$ over the range of 1-20 W below $60 \mathrm{~K}$ even with another factor-of-two scale-down, and this allowed us to reduce the expander microturbine diameter sufficiently to operate at full admission, which would give the efficiency needed for satisfactory cryocooler operation. Optimization of pole-piece contours and dimensions further improve low-load efficiency. Detailed design of the $2 \mathrm{~mm}$ generator was completed in the final report period in conjunction with the overall expander design.

To summarize our study here: we determined that, by far, the best micro-generator approach utilized a solid Nd-B-Fe dipolar rotor core inside a quadrature field winding, as depicted in Figure 15. (We determined the mean thermal coefficient of coercivity for Nd-B-Fe over the

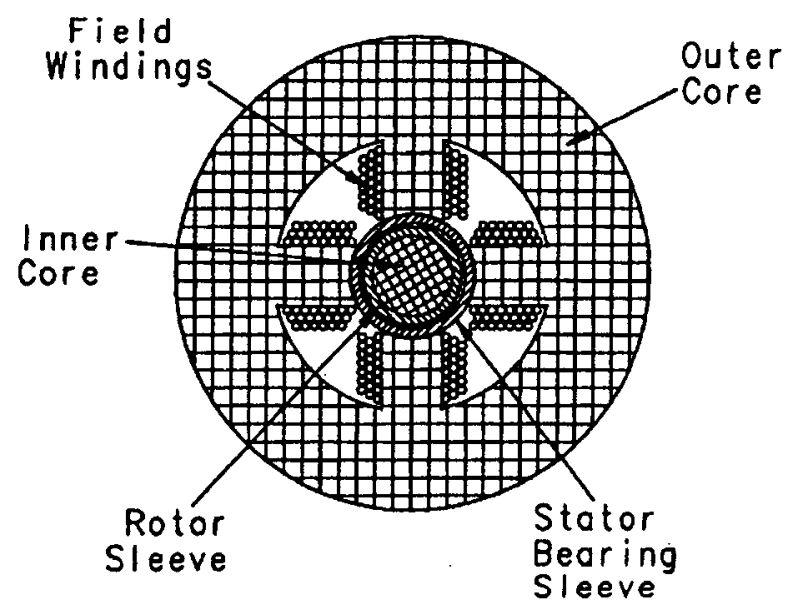

Figure 15. Cross-section of the Magnetic Circuit range of $4 \mathrm{~K}$ to $300 \mathrm{~K}$ to be approximately $+0.05 \% /{ }^{\circ} \mathrm{C}$, compared to a published value of $-0.12 \% /{ }^{\circ} \mathrm{C}$ for the range of 10 $60^{\circ} \mathrm{C}$.) The selected grade (Dexter 1000) of $\mathrm{Nd}-\mathrm{B}-\mathrm{Fe}$ was found to have very stable coercivity $(1000 \mathrm{kA} / \mathrm{m}$ at $\mathrm{RT})$ and acceptable tensile strength $(75 \mathrm{MPa})$ with no significant degradation in either parameter from repeated thermal cycling. Moreover, its mean transverse thermal expansion is (surprisingly) a very close match to that of silicon nitride $(3.5$ $\mathrm{ppm} /{ }^{\circ} \mathrm{C}$ ). Hence, it could be bonded inside the silicon-nitride bearing sleeve, which is strong enough to prevent mechanical failure of the magnetic core at speeds more than three times what otherwise would be possible. The low-perm iron-power field core permits very high efficiency because the high-purity copper winding becomes hyper-conducting at low temperatures. Stator-core hysteresis loss at $6 \mathrm{kHz}$ was $0.3 \mathrm{~W}$ in the $5 \mathrm{~mm}$ rotor and linear with frequency. The winding resistance (each phase) was $0.4 \Omega$ at $77 \mathrm{~K}$, and no-load output was 19 $V_{\mathrm{RMS}}$ at $12 \mathrm{kHz}$. Generator efficiencies between $85 \%$ and $95 \%$ were measured over a wide range of loads and speeds at temperatures from $\sim 80-320 \mathrm{~K}$.

3.4 Compact Recuperators. The Stage 1 MTS design has 800 tubes, each with a $0.8 \mathrm{~mm}$ O.D. and a $0.65 \mathrm{~mm}$ I.D. The first Stage 2 exchangers had 800 tubes, each with a 0.65 $\mathrm{mm}$ O.D. and a $0.4 \mathrm{~mm}$ I.D. While our manufacturing experience with these exchangers is still extremely limited, it appears likely that manifolding costs per module will remain rather high for 
quite some time. This is largely because of the requirement of a precisely fitting flow cage of low axial conductivity surrounding the core and the complexity of manifolding to the shell side without causing significant flow inhomogeneities at the ends.

For small high-effectiveness exchangers, this relatively high manifolding cost per module means there should be no paralleled modules. The number of exchangers required in series is determined primarily by flow inhomogeneity (which is mostly shell-side), as this is the primary limit to effectiveness $\eta_{x}$ at low flow rates - especially with low-conductivity microtubes and helium gas. We did not initially appreciate the severity of the problem of shell-side flow inhomogeneity near the ends, but several experiments led us to believe that this was the primary reason for $\left(1-\eta_{\mathrm{x}}\right)$ being much larger than expected at low flow rates, though blow-by and insufficient spacer grids were also factors.

If flow inhomogeneity can be reduced to $<1.5 \%$, then, with turbulent mixing of the flow between modules, we should be able to achieve the required $99 \%$ effectiveness by using three modules in series in each stage. To obtain a compact arrangement of three long modules inside a cylindrical external dewar, they should stack into an envelop of roughly square cross-section. Since the distance between columns is about $87 \%$ of the distance between rows and some additional space is needed between modules to accommodate the shell-side manifolding, one might suggest that there should be about five or six times as many columns as rows in each module. However, the space required to distribute the shell-side flow uniformly at each end is more than one would initially expect, and it becomes harder to achieve uniform shell-side flow as the number of rows increases. Hence, we chose to go with eight times as many columns as rows, as this appeared to be a reasonable compromise.

The number of tubes per module is largely determined by the maximum practical tube length ( $<500$ times the microtube OD for CuNi alloys) and the required mass flow. The mass flow in the first-stage recuperator is about 1 to $1.3 \mathrm{~g} / \mathrm{s}$ helium. (There are two stages of the smallest practical expander turbines, $0.5-0.7 \mathrm{~g} / \mathrm{s}$.) For effectiveness of $\sim 99 \%$ per stage, we end up needing 800 to 1200 microtubes. Since this seemed to be near a practical manufacturing limit at this point in the process development, we opted for $\sim 800$ microtubes per module (10 rows, 80 columns) initially.

3.4.1 Manufacture. A substantial amount of effort during the past year was devoted to developing improved manufacturing processes for the MTS exchangers. The key is to use a lowthermal-conductivity tube alloy compatible with robust, batch-brazing processes. While the thermal conductivity of Cu-Ni alloy C715 is nearly twice that of SS316, Cu-Ni alloys are far more compatible with fluxless brazing in moderately high vacuum ( 10-100 mTorr) and have much lower outgassing, so $\mathrm{C715}$ will be used in the cryocooler exchangers. However, brazing experiments with stainless microtubes were also performed, as stainless has three clear advantages for closed Brayton power cycles: lower cost, better corrosion resistance, and greater high-temperature strength and stiffness.

The three key manufacturing issues addressed were:

1. Producing the hundreds of tube-holes in the header strips precisely and quickly. 
2. Brazing the tubes into the header-strip with a high-melting-point filler metal such that the tube is sure to be sealed well in the header strip. This involved issues of hole size, header-strip and braze material, and especially the question of where (and in what physical shape) to put the braze alloy around the tube array.

3. Brazing the manifolding and shell-side cages after the tubes have been brazed to the header - without adversely affecting tube/header seal.

After experimentally evaluating laser drilling, blanking, chemical milling, and CNC drilling, it was determined that the combination of chemical milling to slightly under the final hole size followed by CNC drilling was by far the best approach. Chemical milling is a very low-cost option for producing header strips of $\mathrm{Cu}-\mathrm{Ni}$ or stainless alloys $(<\$ 2 /$ header in 200 -piece quantities, after tooling); but when used alone, it is not quite capable of achieving the desired hole precision and straightness. Drilling the holes in a three-piece stack of raw plates on our Dahlih CNC machining center takes $\sim 45$ seconds per hole and requires several tool changes to maintain position accuracy as the drill wear. However, with the holes pre-chemical-milled to $88-98 \%$ of the final size, both the drilling rate and tool life may be increased by a factor of 5 . So item 1 above has been adequately handled for the header strips.

Silver brazing in moderately high vacuum will be used on the $\mathrm{Cu}-\mathrm{Ni}$ header strips with $\mathrm{Cu}$ Ni microtubes because we have shown it to work very effectively in this application with at least three braze alloys, including $\mathrm{BAg}-8$, which is generally considered rather difficult because of its high solidus temperature $\left(780^{\circ} \mathrm{C}\right)$. Because of the enormous potential of stainless steel microtube exchangers in other applications, some effort has also been devoted to optimizing brazing of stainless.

Our experiments suggest that satisfactory fluxless brazing of high-chromium alloys (we tried SS304, SS316L, SS310, Inconel 600, C-276, Haynes C-22, and Haynes-188) is not possible with any palladium-free silver braze alloys, even at $0.1 \mathrm{mTorr}$ (about the highest practical vacuum in most industrial ovens with turbo-molecular pumps) and with the most careful surface preparation. However, we have obtained excellent results with two nickel braze alloys and with Pd-silver braze alloys, both in moderately high vacuum ( $10 \mathrm{mTorr})$ and in several reducing and inert atmospheres. Because of the expense associated with large high-vacuum ovens, we expect it will be necessary to utilize reducing-atmosphere braze processes on stainless exchangers for large, commercial applications in Brayton power cycles.

Several filler-metal fixturing ideas were considered for the vacuum braze process:

a) countersinking the holes in the header-strip for insertion of braze-alloy washers,

b) dip-plating and subsequent reaming of header-strip holes,

c) machining secondary header-strips from the filler material and placing them over the structural header strips.

Option "c" was pursued and tested by first drilling ninety holes (nine rows of ten) in a 0.016"-thick rectangular $\mathrm{Cu}-\mathrm{Ni}$ sheet (representative of the header-strip to be used in the recuperator) and the same number of holes in a similar 0.008 "-thick BAg-8 sheet. Cu-Ni rods of 0.8-mm diameter were trimmed to several inches in length and inserted through the BAg-8 and $\mathrm{Cu}-\mathrm{Ni}$ sheets, with the two sheets laying directly on top of each-other. The rod array then went 
through a second drilled sheet to keep the rods straight. After vacuum brazing, the rods were ground down close to the $\mathrm{Cu}-\mathrm{Ni}$ sheet for inspection of the braze joints around the rod array. Finally, the Cu-Ni sheet was soldered to a closed chamber which was pressurized to check the leak tightness of the rod/sheet braze joints. (See Phase II Proposal for more details). In this way, we tested various hole sizes, number of braze sheets, and orientation of the test unit within the oven during braze. It is important to keep the holes small (no more than one mil of clearance around the rod), to keep the header strips horizontal in the oven, to clean the assembly well prior to brazing, and to use an adequate quantity of braze (two 0.008 " sheets of braze per headerstrip).

The initial brazing experiments were limited to small test pieces that would fit within our Centorr oven. The salient features of a number of more recent experiments are summarized in Tables 3 and 4 . In all cases here, the microtube $O D$ was $0.800 \mathrm{~mm}$ and the holes in the header strip were 0.803 . Except as noted in the comments, the oven ramp rate was $10^{\circ} \mathrm{C} / \mathrm{min}$ to $50^{\circ} \mathrm{C}$, then $25^{\circ} \mathrm{C} /$ min to $200^{\circ} \mathrm{C}$, then $100^{\circ} \mathrm{C} / \mathrm{min}$ to the brazing temperature, soak at the brazing temperature for 5 minutes, then cool at maximum rate (initially, $\sim 100^{\circ} \mathrm{C} / \mathrm{min}$ ). The gas mixtures are all given in volumetric percentages. (See Table 5 for data on the filler metals.) The silver filler material was in the form of metallic foil, while the nickel braze material was in the form of a supple, composite tape of powered metal with polymeric binder.

The manufacturing of the 800 -hole filler-metal $(0.4 \mathrm{~mm}$ foil) strips is now one of the more costly (time consuming) steps, as the high-silver braze alloys are not compatible with standard commercial chemical-milling processes. Hence, they were drilled on a CNC machine. Of course, these braze alloys drill much more readily than the $\mathrm{Cu}-\mathrm{Ni}$ or stainless alloys, but they still require about 10 seconds per hole, though this process can probably be further optimized. We have very recently found a vendor who can perform quality chemicl-milling of high-silver braze alloys. We have not yet determined whether or not chemical-milling processes are compatible with nickelbraze alloys. In production, these filler-metal strips could be punched at very low cost per part, but the die cost is not justified during the development and prototyping stage.

With Cu-Ni tubes and header-strips brazed together using eutectic BAg-8, subsequent torch brazing of manifolding and cages may be done sequentially with lower melting brazes more suited to torch brazing (BAg-10, BAg-7) without complex fixturing.

Stainless alloy $316 \mathrm{~L}$ was selected for the manifold and cage material for four reasons:

1. Compared to Cu-Ni, stainless cages offer better dimensional stability and hence better control of the gap distance between the inside edges of the cage and the outer rows of tubes. Control of this gap will be important for good shell-side flow homogeneity.

2. Compared to $\mathrm{Cu}-\mathrm{Ni}$, thinner material may be used for stainless cages due to higher strength and so lower thickness and conduction loss is achieved.

3. Compared to other steels, its coefficient of thermal expansion matches Cu-Ni's very well.

4. Compared to most other stainless steels, it has lower outgassing.

Over the past year, a number of 800-tube MTS recuperator modules have been satisfactorily brazed and tested of three designs: (A) the preliminary design as proposed in the 
Phase II proposal; (B) a first revision to simplify some manufacturing difficulties in the manifolding, and $(\mathrm{C})$ a second, major revision to improve shell-side flow distribution for improved effectiveness, as described in the following section.

\begin{tabular}{|c|c|c|c|c|c|c|}
\hline \multicolumn{7}{|c|}{ Table 3. Micro-tube array brazing experiments. } \\
\hline Test \# & 1 & 2 & 3 & 4 & 5 & $6 \mathrm{~b}$ \\
\hline Tube alloy & $\mathrm{C} 715$ & $\mathrm{C} 715$ & $\mathrm{C} 715$ & $\mathrm{SS} 316$ & $\mathrm{SS} 316$ & $\mathrm{SS} 316$ \\
\hline Header-strip alloy & $\mathrm{C} 715$ & $\mathrm{C} 715$ & $\mathrm{C} 715$ & $\mathrm{SS} 316$ & $\mathrm{SS} 316$ & $\mathrm{SS} 316$ \\
\hline Surface prep. method & 1 & 1 & 1 & 1 & 1 & 1 \\
\hline Braze alloy & $\mathrm{BAg}-8$ & $\mathrm{BAg}-8$ & $\mathrm{BAg}-8$ & $\mathrm{BNi}-2$ & $\mathrm{BNi}-2$ & $\mathrm{BNi}-2$ \\
\hline Braze foil thickness (mm) & 0.4 & 0.4 & 0.4 & 0.25 & 0.25 & 0.25 \\
\hline Braze foil location & top & top & top & top & top & top \\
\hline Initial (Cold) Purge Gas & $\mathrm{N}_{2}$ & $5 \mathrm{H}_{2}-95 \mathrm{Ar}$ & $5 \mathrm{H}_{2}-95 \mathrm{~N}_{2}$ & $\mathrm{~N}_{2}$ & $5 \mathrm{H}_{2}-95 \mathrm{Ar}$ & $50 \mathrm{H}_{2}-50 \mathrm{Ar}$ \\
\hline Brazing gas & $\mathrm{N}_{2}$ & $5 \mathrm{H}_{2}-95 \mathrm{Ar}$ & $5 \mathrm{H}_{2}-95 \mathrm{~N}_{2}$ & - & $5 \mathrm{H}_{2}-95 \mathrm{Ar}$ & $50 \mathrm{H}_{2}-50 \mathrm{Ar}$ \\
\hline Brazing gas flow rate, CFH & 5 & 15 & 15 & - & 15 & 20 \\
\hline Brazing vacuum, mTorr & - & - & - & 500 & - & - \\
\hline Brazing temperature, ${ }^{\circ} \mathrm{C}$ & 820 & 820 & 820 & 1050 & 1050 & 1050 \\
\hline Tube surface quality & poor & good & good & good & poor & v. good \\
\hline Braze wetting/flow out & poor & $\mathrm{v}$. good & good & $\mathrm{v}$. good & poor & good \\
\hline Joint/fillet quality & poor & $\mathrm{v}$. good & good & v. good & poor & v. good \\
\hline Test \# & 1 & 2 & 3 & 4 & 5 & $6 \mathrm{~b}$ \\
\hline
\end{tabular}

Surface prep. method 1: Soapy water, then $\sim 5 \% \mathrm{H}_{2} \mathrm{SO}_{4}$, then dry in nitrogen.

Table 3 Comments: Test 3: Several joints were dry, and the failure of the filler metal to flow into them appeared to have been caused by minute burrs around the holes.

Test 6b: A dark green oxide film, uniform and adherent, formed over the stainless parts during brazing, but it was readily removed by sulfuric acid and did not appear to affect braze action, suggesting it had formed slowly and after the liquidus was reached. The first attempt under similar conditions apparently did not provide adequate gas coverage of the parts during brazing and several joints failed. An internal oven chamber to improve gas confinement was then added. 


\begin{tabular}{|c|c|c|c|}
\hline \multicolumn{5}{|c|}{ Table 4. Micro-tube array brazing experiments. } \\
\hline Test \# & 7 & 8 & 9 \\
\hline Tube alloy & $\mathrm{SS} 316$ & $\mathrm{SS} 316$ & $\mathrm{SS} 316$ \\
\hline Header-strip alloy & $\mathrm{SS} 316$ & $\mathrm{SS} 316$ & $\mathrm{SS} 316$ \\
\hline Surface prep. Method & 1 & 1 & 1 \\
\hline Braze alloy & $\mathrm{BNi}-2$ & $\mathrm{BNi}-5$ & $\mathrm{BNi}-5$ \\
\hline Braze foil thickness (mm) & 0.25 & 0.25 & 0.5 \\
\hline Braze foil location & top & top & top \\
\hline Initial (Cold) Purge Gas & $5 \mathrm{H}_{2}-95 \mathrm{~N}_{2}$ & $50 \mathrm{H}_{2}-50 \mathrm{Ar}$ & $50 \mathrm{H}_{2}-50 \mathrm{Ar}$ \\
\hline Brazing gas & $5 \mathrm{H}_{2}-95 \mathrm{~N}_{2}$ & - & $50 \mathrm{H}_{2}-50 \mathrm{Ar}$ \\
\hline Brazing gas flow rate, CFH & 20 & - & - \\
\hline Brazing vacuum, mTorr & - & 100 & - \\
\hline Brazing temperature, ${ }^{\circ} \mathrm{C}$ & 1050 & 1175 & 1125 \\
\hline Tube surface quality & poor & poor & v.good \\
\hline Braze wetting/flow out & poor & poor & fair \\
\hline Joint/fillet quality & poor & poor & fair \\
\hline Test \# & 7 & 8 & 9 \\
\hline
\end{tabular}

Table 4 Comments:

Test 7: Again, a similar dark green oxide film formed.

Test 8: The surfaces were covered by a uniform, adherent black oxide. An extra 30minute soak was included at $850^{\circ} \mathrm{C}$ to improve vacuum. This was not beneficial.

Other brazing experiments on SS310, Inconnel 600, and Haynes C-22 parts gave very promising results with Palcusil-25 in $5 \mathrm{H}_{2}-95 \mathrm{Ar}$. Pure silver and $\mathrm{BAg}-23$ were not satisfactory in $5 \mathrm{H}_{2}-95 \mathrm{Ar}$ on any high-chromium alloy, but still need to be evaluated in $50 \mathrm{H}_{2}-50 \mathrm{Ar}$ and pure $\mathrm{H}_{2}$.

\begin{tabular}{|c|c|c|c|c|c|c|c|c|c|}
\hline \multicolumn{10}{|c|}{ Table 5. Some Common Braze Alloys. } \\
\hline $\begin{array}{c}\text { Braze } \\
\text { Alloy }\end{array}$ & $\begin{array}{c}\text { solidus } \\
{ }^{\circ} \mathrm{C}\end{array}$ & $\begin{array}{c}\text { liquidus } \\
{ }^{\circ} \mathrm{C}\end{array}$ & $\begin{array}{c}\mathrm{Ni} \\
\%\end{array}$ & $\begin{array}{c}\mathrm{Cr} \\
\%\end{array}$ & $\begin{array}{c}\mathrm{Si} \\
\%\end{array}$ & $\begin{array}{c}\mathrm{Cu} \\
\%\end{array}$ & $\begin{array}{c}\mathrm{Ag} \\
\%\end{array}$ & $\begin{array}{c}\text { Sn } \\
\%\end{array}$ & $\begin{array}{c}\text { Other } \\
\text { (Typical) }\end{array}$ \\
\hline BNi-5 & 1080 & 1135 & 70 & 19 & 10 & & & & \\
\hline BNi-2 & 990 & 1000 & 82 & 7 & 4 & & & & $3 \mathrm{~B}, 3 \mathrm{Fe}$ \\
\hline Palsil 10 & 1002 & 1065 & & & & & 90 & & $10 \mathrm{Pd}$ \\
\hline BAg-23 & 960 & 970 & & & & & 85 & & $15 \mathrm{Mn}$ \\
\hline PalCuSil25 & 900 & 950 & & & & 54 & 21 & & $25 \mathrm{Pd}$ \\
\hline
\end{tabular}




\begin{tabular}{|c|c|c|l|l|l|c|c|c|c|}
\hline CuSil-1 & 775 & 806 & & & & 34 & 63 & 1 & $1.8 \mathrm{Ti}$ \\
\hline BAg-8 & 779 & 779 & & & & 28 & 72 & & \\
\hline BAg-10 & 690 & 740 & & & & 20 & 70 & & $10 \mathrm{Zn}$ \\
\hline BAg-18 & 602 & 718 & & & & 30 & 60 & 10 & \\
\hline InCuSil & 605 & 715 & & & & 27 & 59 & & $12.5 \mathrm{In}, 1.3 \mathrm{Ti}$ \\
\hline BAg-7 & 618 & 652 & & & & 22 & 56 & 5 & $17 \mathrm{Zn}$ \\
\hline HMP & 304 & 310 & & & & 0.1 & 2.2 & 0.1 & $97.5 \mathrm{~Pb}, .2 \mathrm{Sb}, .1 \mathrm{Bi}$ \\
\hline Sn-96 & 221 & 221 & & & & & 3.5 & 96.2 & $.05 \mathrm{~Pb}, 05 \mathrm{Sb}, .05 \mathrm{Bi}$ \\
\hline $63-37$ & 183 & 183 & & & & 0.02 & & 62.5 & $37 \mathrm{~Pb}, .2 \mathrm{Sb}, 1 \mathrm{Bi}$ \\
\hline
\end{tabular}

3.4.2 Compact Shell-side Manifolding for High Homogeneity of flow. If the total exchanger volume is to be minimized, all plumbing required for the series manifolding and interconrrections must be limited to the ends of the modules (not extend beyond the header width), as the module length is about three times its width. If only three modules are being stacked in the thickness (row) direction, the shell-side manifolding could add quite a bit to the total thickness with little effect on total external dewar ID, though we'll to limit this overhang as much as practical for other possible applications requiring more modules.

The tube-side manifolding is simplified because the viscous pressure drop in the microtubes under typical conditions is large compared to the dynamic head inside the header cap. For example, the pressure drop in the middle module of the first-stage recuperator $(\sim 200 \mathrm{~K})$ at about $0.8 \mathrm{mg} / \mathrm{s}$ per microtube $(0.63 \mathrm{~mm} \mathrm{ID,} 0.8 \mathrm{~mm} \mathrm{OD})$ is about $1200 \mathrm{~Pa}$. The mean velocity in the microtubes is $\sim 3 \mathrm{~m} / \mathrm{s}$, and the mean transverse mach number within the header cap is $\sim 0.003$, corresponding to a mean dynamic head variation of $\sim 2 \mathrm{~Pa}$ at each end (though it can be more than an order of magnitude greater near the header inlets, so a porous deflector plate is beneficial right at the inlet). Hence, tube-side flow homogeneity is primarily dependent on the precision of the microtube ID.

For the shell-side flow, on the other hand, the longitudinal pressure drop for the above module is only $\sim 200 \mathrm{~Pa}$ within the core for tube center-to-center spacing of $1.6 \mathrm{~mm}$ (ignoring the effects of the ten spacer grids, which might be substantial). Even with ten spacer grids along the tube length, it is unlikely that rms spacing errors will be less than $0.005 \mathrm{~mm}$. Reducing the spacing to increase the longitudinal pressure drop (in an attempt to reduce the effects of the inlet dynamic head) is likely to only increase manufacturing difficulties. The spacer grids are actually found to help considerably in homogenizing the flow - as long as we are careful to minimize blowby around their perimeter by maintaining close spacing to the cage. (Note that they are secured to the cage by tabs protruding through slits in washers which are soldered over relatively large holes in the cage after assembly to facilitate alignment. These details are not shown in the current drawings.) 


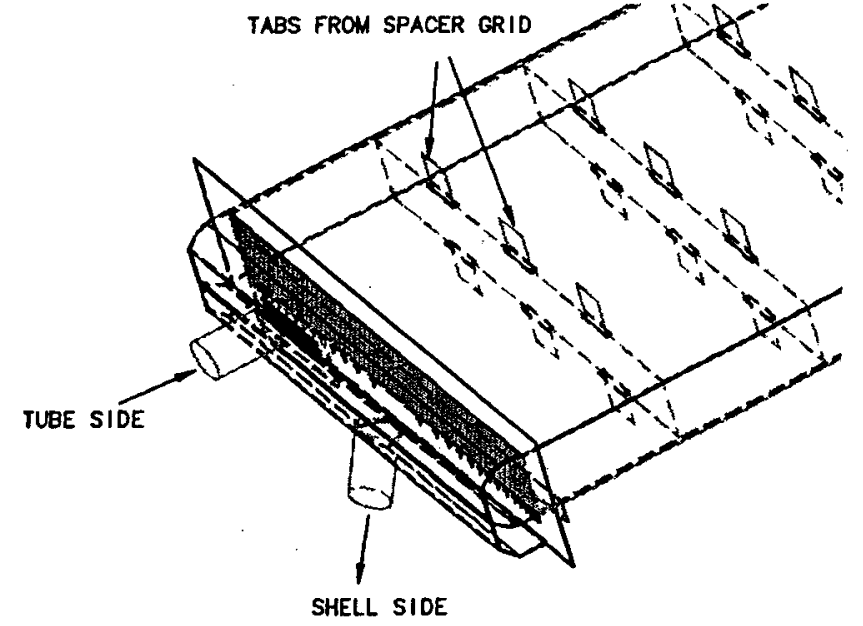

Figure 16. Tube/shell headers on exchanger module.

To achieve $99 \%$ flow homogeneity throughout most of the core, it would appear that the shell-side manifolding must distribute the flow uniformly across the full width of the module such that this flow enters the core (at the end) with rms mach number variations below 0.003 . The main shell-side feed should come in from the ends, near the center, just above or below the tube side feed. Figures 16-18 illustrate the revised module, which is no longer intended to be compatible with parallel manifolding.
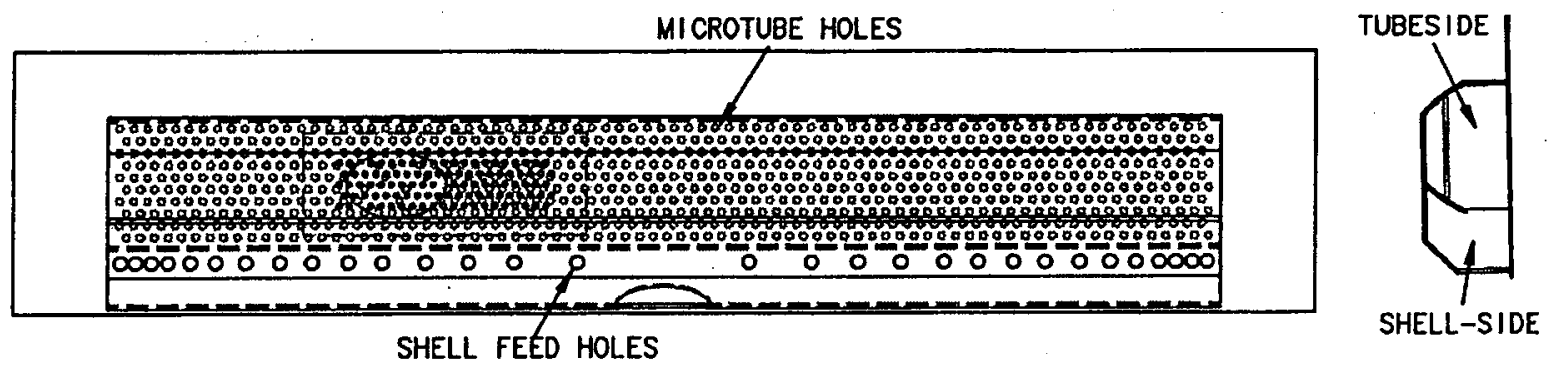

Figure 17 above, Tube/shell header section views.

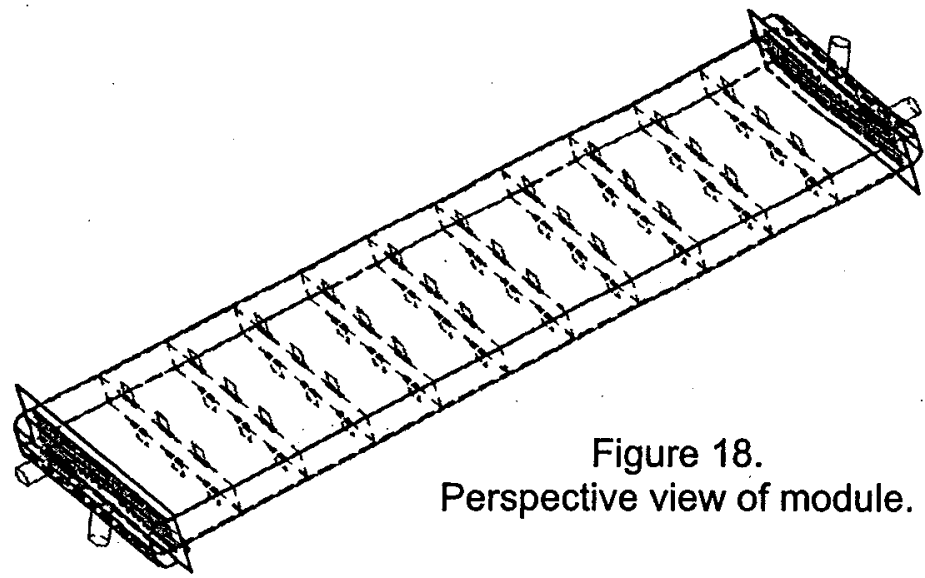

As illustrated, we now use two "header caps" at each end - a tube-side header cap in the normal sense, and an adjacent shell-side manifold cap that distributes the shell-side flow uniformly across the full width of the module. Since the shell-side inlet manifold pressure decreases with transverse distance away from the main feed line, the size or density of the shell-side feed holes (from the shell-side cap to the cage) must increase toward the edges of the header strip. An initial feed-hole distribution was determined experimentally.

Initial experimental tests on nitrogen, as summarized in Table 6, on an 800-tube module of the new design (with our first-pass estimate for the feed holes) confirmed the dramatically improved shell-side flow uniformity. The measured effectiveness $\eta_{x}$ is compared to the 
calculated effectiveness - first assuming zero external heat leak $\left(\eta_{\times 0}\right)$ and then with the $\sim 2.5 \mathrm{~W}$ experimental external heat leak included $\left(\eta_{\mathrm{x}_{\mathrm{L}}}\right)$. Here, shell-side flow non-uniformity appears to be $<1.5 \%$. After a few simple experiments, a more optimum feed-hole distribution was determinined that now permits over $99 \%$ effectiveness in a single helium module with negligible increase in shell-side pressure drop.

It should be noted that the original concept of paralleled modules for high-capacity exchangers at somewhat lower effectiveness did not have a problem with uniform distribution of shell-side flow at the inlet, as velocities were very low throughout the large, inlet manifold cavity. However, that approach was not well suited to series connections of single modules. Moreover, it is possible that a variation on the new approach would also simplify the manufacturing difficulties associated with paralleling modules, as the prior approach did not easily permit individual module testing prior to paralleling, and the final brazing operations were quite complex.

\begin{tabular}{|c|c|c|c|c|c|}
\hline \multicolumn{6}{|c|}{ Table 6: Exchanger 1 Test Results with Nitrogen Gas } \\
\hline & Units & & & & \\
\hline Vol. Flow & SCFH & 32 & 64 & 122 & 250 \\
\hline $\mathrm{N}_{2}$ Mass Flow & $\mathrm{g} / \mathrm{s}$ & 0.45 & 0.9 & 1.8 & 3.75 \\
\hline Tube-S In, T & $\mathrm{K}$ & 292.6 & 293.8 & 293.9 & 292.9 \\
\hline Shell-S In, T & $\mathrm{K}$ & 123.3 & 126.5 & 132.1 & 125.8 \\
\hline Shell-S Out, T & $\mathrm{K}$ & 279.4 & 280.1 & 280.1 & 280.5 \\
\hline Exchange P & $\mathrm{W}$ & 56 & 112 & 220 & 460 \\
\hline Experimental $\eta_{\mathrm{X}}$ & $\%$ & 89 & 91 & 92 & 91 \\
\hline Calc. $\eta_{\mathrm{Xo}}$ & $\%$ & 93 & 94 & 93.4 & 90 \\
\hline Calc. $\eta_{\mathrm{XL}}$ & $\%$ & 89 & 94 & 93 & 92 \\
\hline
\end{tabular}

3.4.3 Tube-side Homogeneity and Subchannel Coupling. The experience with shell-side flow problems in Stage-1 exchangers gave us insight for the Stage-2 exchangers. For the initial Stage-2 exchangers, the exchanger manifolding was designed as outlined (for the final Stage-1 exchangers) in the previous subsection 3.4.2. Even so, the performance of this first generation of Stage-2 exchangers was poor.

At a given pressure drop, laminar flow through a tube is proportional to I.D. to the fourth power. As Stage-2, MTS-exchanger tubes have such small I.D.'s, even a 0.0004" I.D. variation will create a $10 \%$ variation in tube-side flow! This problem contributed to the unexpected poor performance of the initial Stage-2 exchangers. An effort was undertaken to sort the tubes based on carefully measured flow rates, in part to assess the impact of this tube-side inhomogeneity. A semi-automated system was set-up to move a cup under a stream of water coming through the tube under test. Pressure was tightly controlled with ballast vessel. Each tube had to be inserted 
into the seal by hand, so the process of obtaining eight hundred tubes having flow-rate variations $<2 \%$ was very labor intensive.

Another Stage-2 MTS performance issue was the excessively small center-to-center tube spacing. The initial design had a spacing of only $1.12 \mathrm{~mm}$. As the spacer grids would accept gage pins of $0.82 \mathrm{~mm}$, the location of each tube can vary from nominal by $\pm 0.09 \mathrm{~mm}$, and the area of the shell-side subchannels (the individual flow area between tubes, each surrounded by three tubes) can vary by a factor of 1.6 from minimum to maximum. In addition, the small space between tubes reduces the coupling between subchannels. This coupling allows mass-flow and heat transfer perpendicular to the primary mass-flow direction. Temperature and pressure gradients are better normalized with more complete subchannel coupling - giving better shellside flow homogeneity.

A new generation of Stage II heat exchanger was designed and procured just prior to the end of Phase II. The design has larger tube spacing and tighter control over tube spacing. Manufacture was started on this unit and will continue as part of our ongoing Phase III heat exchanger development. The performance characteristics will give us valuable experimental information about the impact of the effects discussed in the last paragraph. Depending upon this exchanger's performance, we may then build a unit with tubes sorted by flow-rate as described above.

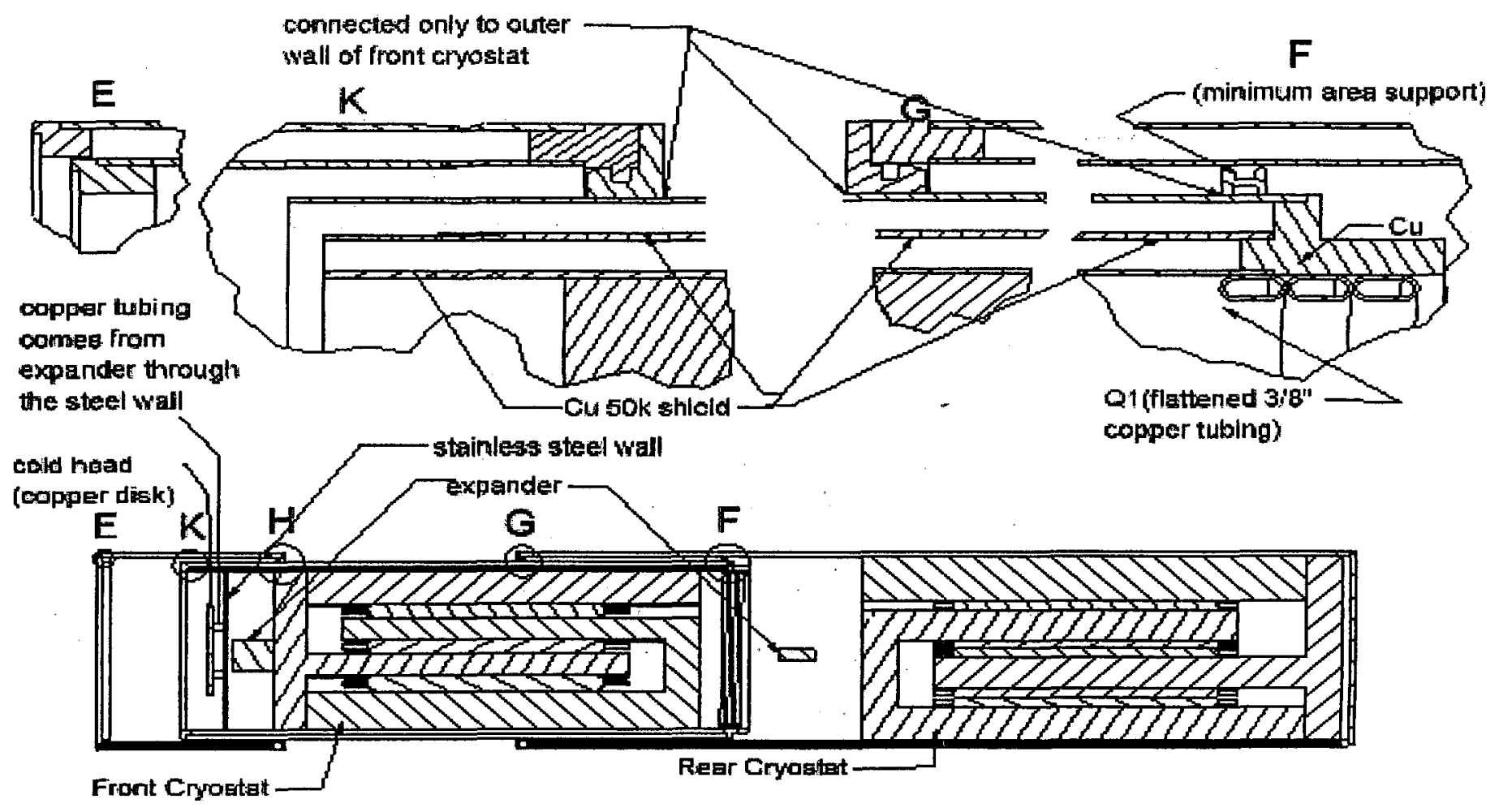

Figure 19, Cross-sectional View of Cryostat Design 
3.5 Overall System. Conceptual design work on the overall system has progressed well beyond that of the Phase I. Spacing, heat transfer issues, and cryostat assembly issues were largely addressed during the final report period. A fairly detailed conceptual design is complete; the next step in the design would be to develop the parts that are in the CAD model to final dimensions, and draft the components. See Figures 19 and 20 for system design. Vendors were found for the cryostat walls, and our experience with compact vacuum and insulating systems would allow us to braze and assemble the system in-house.

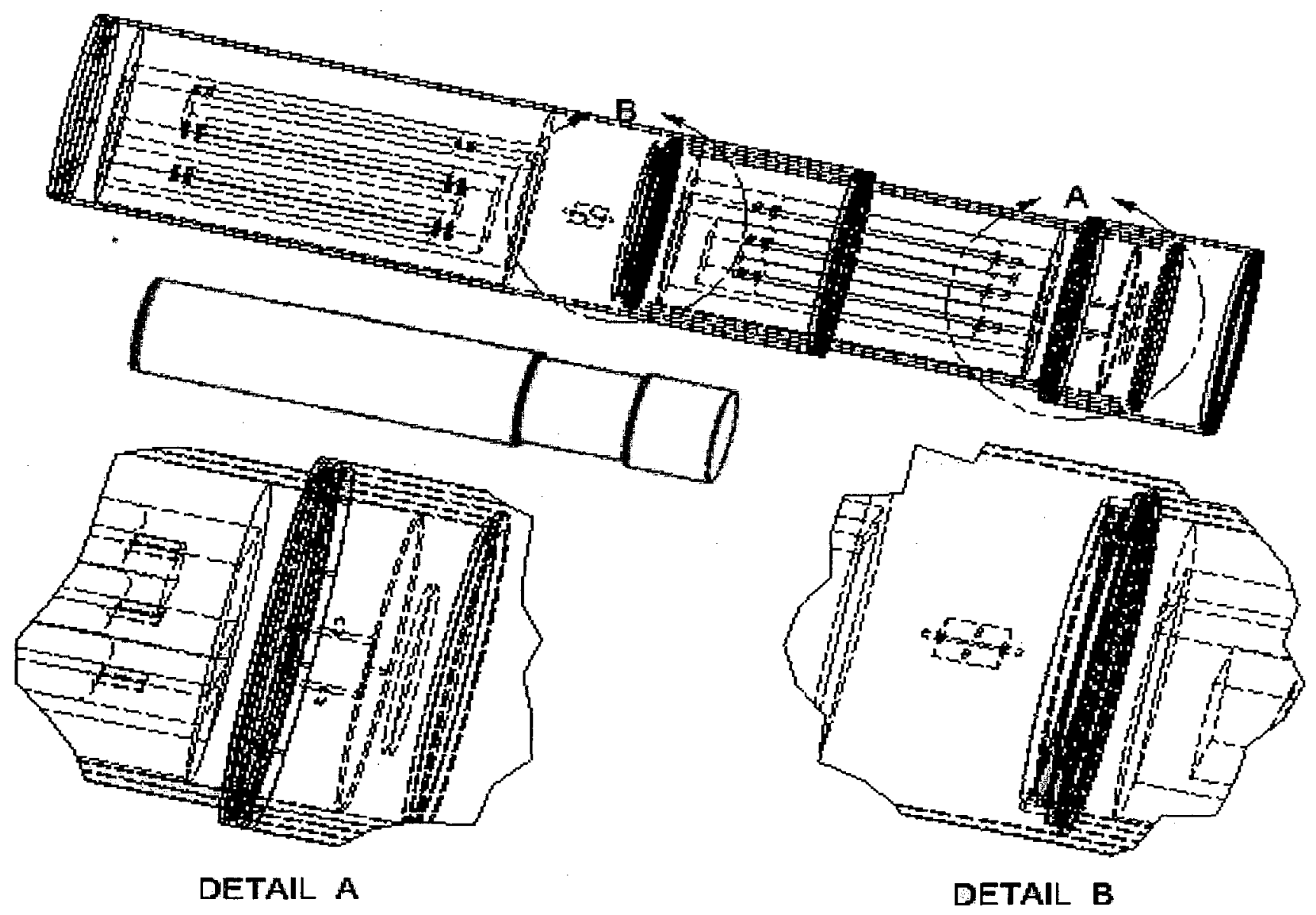

Figure 20, 3-D Perspective Views of Cryostat Design

During the Phase II, we developed a detailed computer model of the CBC with MTS heat exchangers, our parametric turbine model, and the preliminary model of the RBC with MTS heat exchangers. The model allows the input of several dozen engineering and design parameters, from which all pressure drops, exchanger masses, temperature differences, powers, efficiencies, specific masses, etc. are calculated.

The system design is still expected to be very much as outlined in the Phase II proposal except that a four-stage centrifugal compressor will be used and the mass flows will be a little higher. The original design is shown in Figure 1. The four-stage design is required because the compressor CFD simulations show that higher rotational rates are needed than the initial estimates predicted and because long-term rupture strength of the titanium beta alloys is much 
less than was expected from short-term yield-strength data above $120^{\circ} \mathrm{C}$. From the expander experimental work, it has become clear that leakage from the turbine inlet plenum to the axial bearing region cannot be kept as low as expected, even with the scale-down in bearing size. Thus, to obtain acceptable system efficiency, the expander flow-rates would increase by perhaps $50 \%$, but the RBC may still be impractical at this size. In fact, it could be that the smallest practical RBC expander would utilize a pair of $7 \mathrm{~mm}$ expander turbines with a $5 \mathrm{~mm}$ gas bearing. This translates to a minimum mass flow rate of $\sim 5 \mathrm{~g} / \mathrm{s}$ for the $10 \mathrm{~K}$ expanders and bearing - an order of magnitude larger than initially projected.

To be sure, it is certainly possible to scale down to under $1 \mathrm{~g} / \mathrm{s}$, and it should be possible to obtain efficiency that is competitive with the best alternative options for $2 \mathrm{~W}$ at $10 \mathrm{~K}$. However, the tolerance requirements and manufacturing difficulties at this size would appear to make a $2 \mathrm{~W}$, $10 \mathrm{~K}$ RBC cryocooler so expensive as to be impractical. Increasing the expander and bearing dimensions by a factor of two and the cooling power by a factor of five dramatically reduces the expander cost, largely because well established manufacturing and inspection processes are then adequate. The first stage $(50 \mathrm{~K})$ expander should be practical at $\sim 3 \mathrm{~g} / \mathrm{s}$. Thus, the compressor would need to have a capacity of $\sim 10 \mathrm{~g} / \mathrm{s}$. We have not tried to determine whether or not there are substantial applications for $10 \mathrm{~W}, 10 \mathrm{~K}$ cryocoolers with ultra-low vibration. 


\section{Update of Initial Phase III Efforts}

4.1 Compact Recuperators. Because of its very low mass, some interest in the MTS is developing in the cryogenics community. An MTS presentation was given the NASA 2001 Space Cryogenics Workshop. Several companies have expressed a desire to purchase custom, hand-built units at premium prices. The upcoming issue of Cryogenics will include a technical paper by Boman and Doty outlining the cryogenic issues of the MTS in theory and (primarily) manufacturing. At this phase of our development, hand-assembled units are all that we have available. This dramatically limits the marketing potential of the MTS for two reasons:

1. The high production cost associated with hand assembling the tubes within the headers and with hand assembling and brazing/soldering the manifolding and cage pieces.

2. The upper temperature limit that is imposed by torch brazing.

Now that the MTS HX design and manufacture has developed to this point, the next generation MTS is being developed for Phase III with three specific goals in mind. One is to make another major improvement in shell-side flow uniformity. A second goal is to drastically reduce manufacturing costs by developing manifolding and shell-side cages that are compatible with an oven-brazing process. Said development is also critical to the third goal, which is to create a manufacturing process that will allow MTS HX's made of higher temperature materials and brazes, ultimately superalloy construction.

Various non-cryogenic applications are being examined for MTS exchangers. In particular, gas-turbine power generation is a very promising application. Also, pebble bed modular (nuclear) reactors (PBMR's) should benefit from a high-performance, gas-to-gas recuperator that promises to be more cost-effective for high-temperature, high-pressure applications.

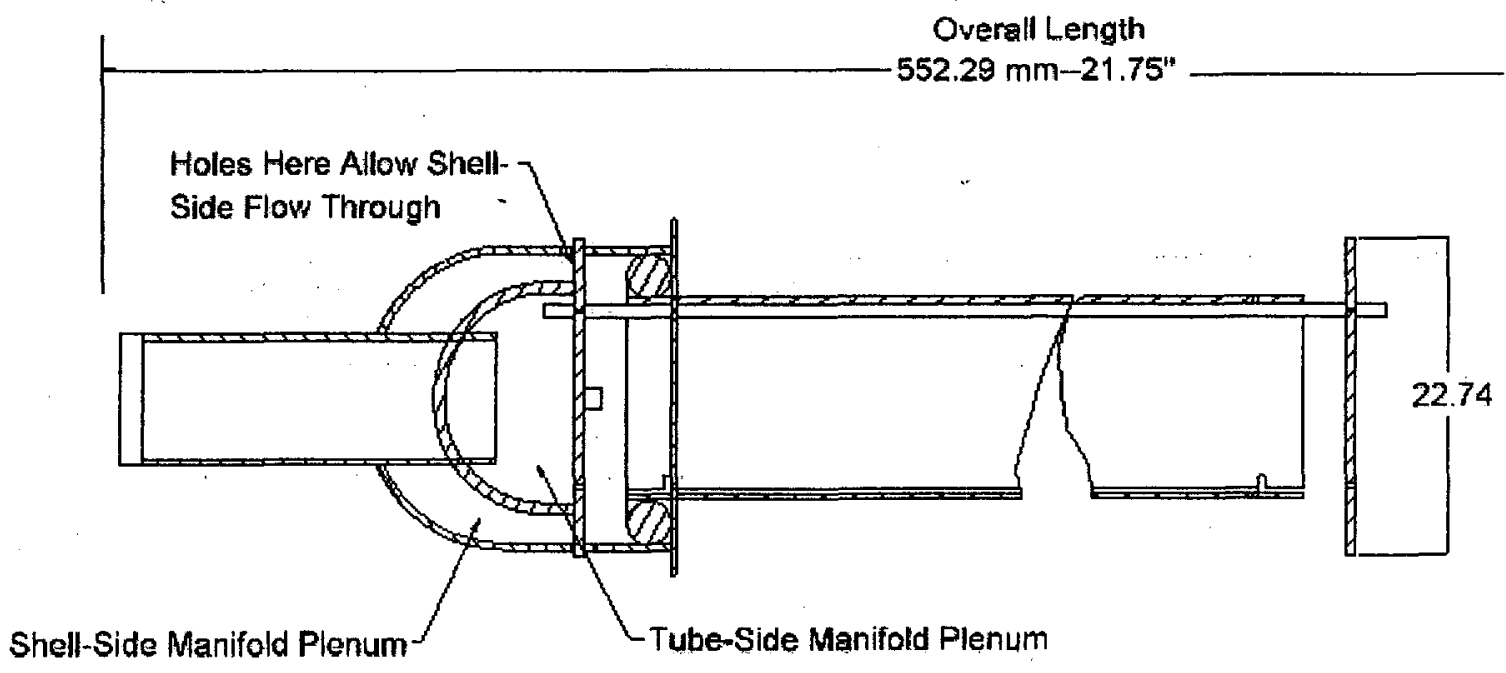

Figure 21 : Section view of next generation design. 
Detailed design, procurement, and fixturing is complete for the first prototype run of Phase-III, stainless-steel exchangers. Key features of the new design include a new manifold configuration that allows the shell-side flow to enter from the top and bottom simuitaneously (see Figure 21). It also allows better heat-transfer between the two fluid streams while they are in the manifold region and batch brazing of the manifolding. The new shell design is also compatible with batch brazing and larger volume production. Mechanical joints hold the shell completely together before any braze has been applied. A modified spacer-grid "hole" profile provides more shell-side flow area around the outside of the tubes and should reduce shell-side flow resistance, which will decrease pressure-drop (Figure 23). Other changes include longer tubes (from 37.6 $\mathrm{cm}$ in the current units to $47.8 \mathrm{~cm}$ in the next generation design) and better control over tube spacing.

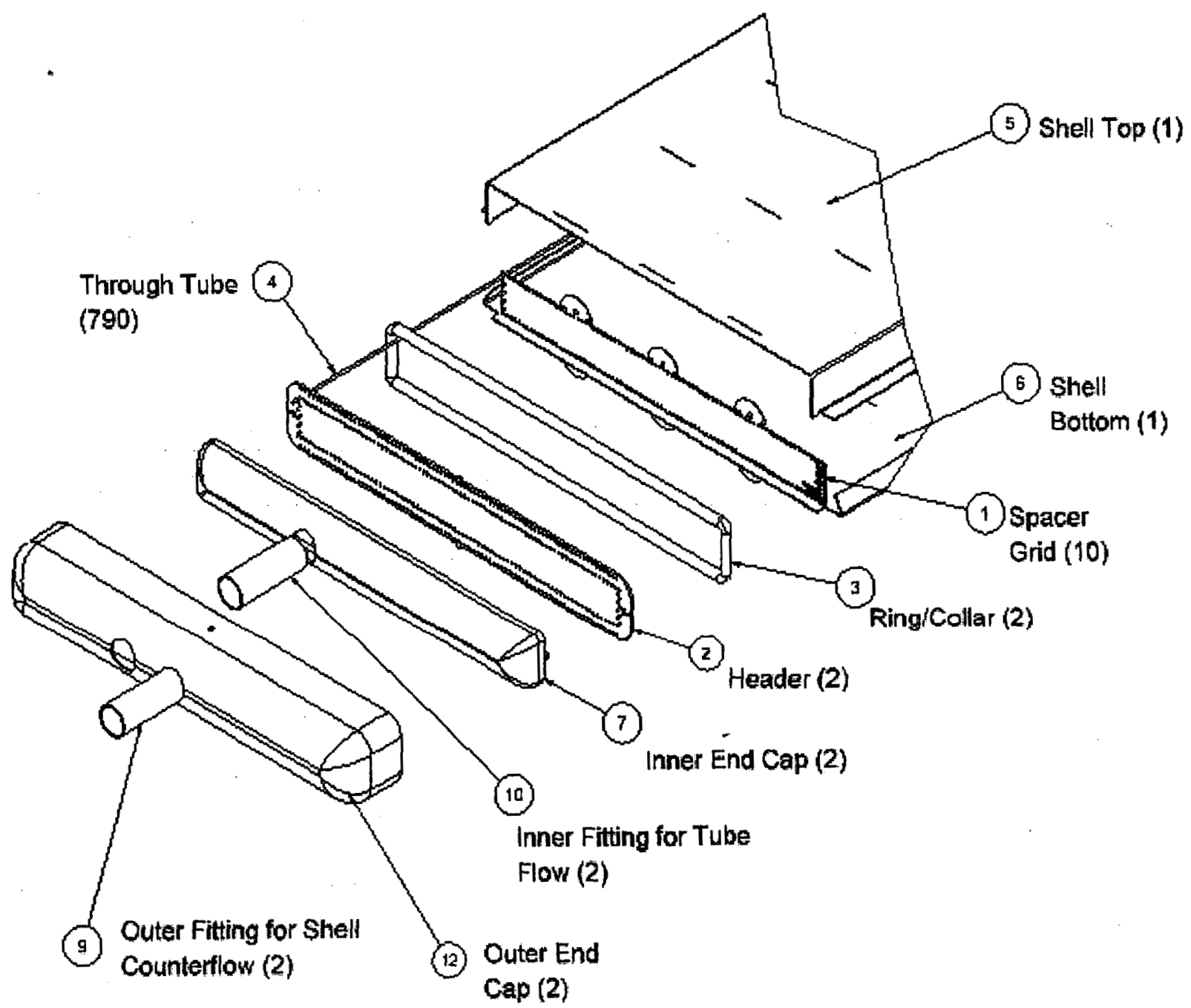

Figure 22 : Exploded view of next generation design 


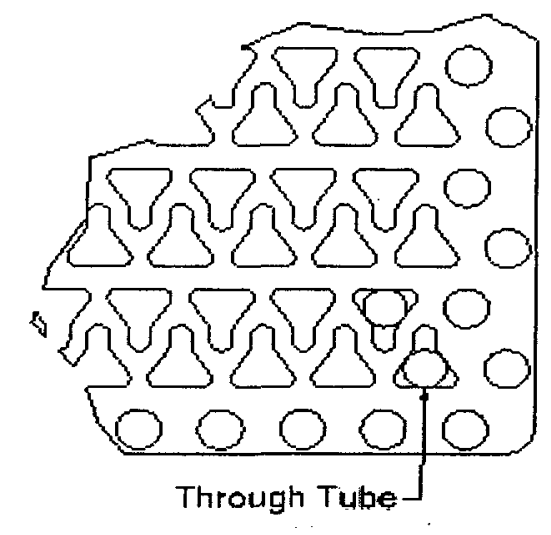

Figure $23:$ Close-up of next generation spacer grid pattern.

If batch brazing can become standardized enough for production, the next step will be to develop fixtures that will enable either automation of tube insertion through a Gattling type arrangement, or a substantial increase in the hand assembly rate. There remains much work in the maturation of the heat exchangers as a product, and, although the technical goals in the following-on funding commitment were not quite met, Doty Scientific is going forward with the commercialization effort.

4.2 Micro-turbine Expanders. To date, two companies have expressed interest in four- and five-mm, cryogenic micro-turbine expanders as built by Doty Scientific. Though these expander sizes were, finally, not appropriate for a $1 \mathrm{~W}, 10 \mathrm{~K}$ cryocooler; the micro-turbine and micro-generator development work occurring in Phase II may make them appropriate for other cryogenic units. If enough interest is shown, this work is likely to continue solely on a component basis.

Integral to a working expander is, of course, a micro-generator. But the benefits of Phase-I and -II developments there are not limited to the gas-bearing, micro-turbine expander. Currently, a search of the literature is being done to assess the state of the art in this area of MEMS (micro-electromechanical systems) technology. A technical paper outlining the analysis and manufacture of the two generators is planned. We intend to also report the material properties of Nd-B-Fe that were determined during Phase I (see section 3.3).

Other non-cryogenic commercial applications are being developed for the $2 \mathrm{~mm}$ expander. These efforts were launched by the $2 \mathrm{~mm}$ cryo-spinner that was designed, procured, and partially manufactured during Phase II. The primary market is expected to be an ultra-highspeed NMR sample spinner. A Phase-I grant has just been awarded to the PI by the National Institutes of Health for development of such a sample spinner. The goal for this spinner is 50 $\mathrm{kHz}$. Accomplishment would be a dramatic step forward in molecular structure determination of insoluble helical membrane proteins that are critical to biological function.

Another potential application spurred by Phase II efforts is the micro-gas-turbine engine. This outgrowth development-work represents a sizeable benefit of the DOE investment. In addition to the expander and heat exchanger development, CFD and system-analysis software maturation during Phase II is very useful here. Specifically, a grant proposal was recently submitted to the United States Army in response to their solicitation for low-power, low-mass, fieldable generators for the dismounted soldier and for remote, military robots. In general, our work on the Brayton Power Cycle (which could revolutionize small-scale and on-site power generation) has been given a significant lift from much of the Phase II effort. 


\section{References}

1. Walker, Miniature Refrigerators for Cryogenic Sensors and Cold Electronics, Clarendon Press, Oxford, 1989.

2. D. B. Mann, H. Sixsmith, W. A. Wilson, and B. W. Birmingham, "A Refrigeration System Incorporating a Low-capacity, High-speed, Gas-bearing-supported Expansion Turbine", in Adv. in Cryo. Engr., Vol. 8, Plenum Press NY (1962) 221-227.

3. H. Sixsmith and W. L. Swift, "A pair of miniature helium expansion turbines", in Adv. in Cryo. Engr., Vol. 27, Plenum Press, NY (1982) 649-655.

4. V. lannello and H. Sixsmith, "Magnetic bearings for cryogenic turbomachines", in Adv. in Cryo. Engr., Vol. 37 B, Plenum Press, NY (1992), 809-816.

5. K. D. Timmerhaus, "Cryogenic Process Engineering", Encycl. of Phys. Sci. and Tech., Vol. 4, Academic Press, 1992.

6. J. D. Fuerst, "Experience with Small Turbomachinery in a 400 W Refrigerator", in Adv. in Cryo. Engr., Vol. 41 A, 949-955, Plenum Press, NY (1992).

7. H. Sixsmith, R. Hasenbein, and J. A. Valenzuela, "A Miniature Wet Turboexpander", in Adv. in Cryo. Engr., Vol. 35 B, Plenum Press, NY (1990) 989-995.

8. K.J. Yang, H.B. He, G. Ke, and G.Y. Li, "Application and Test of Miniature Gas Bearing Turbines", in Adv. in Cryo. Engr., Vol. 35 B, Plenum Press, NY (1990) 997-1003.

9. F. D. Doty and P. D. Ellis, Rev. Sci. Instrum., 52 (12), "Design of High Speed Cylindrical NMR Sample Spinners", (1981) 1868-75.

10. F. D. Doty, "Probe Design and Construction", Encycl. of NMR, Vol. 6, Wiley, 1996.

11. F. D. Doty, L. G. Hacker, and J. B. Spitzmesser, "Supersonic Sample Spinner", U. S. Pat \# 5,508,615, 1996.

12. F. D. Doty, "Solid State Probe Design", Encyclopedia of NMR, Vol. 7, Wiley, 1996.

13. T. Kato et al, "A Large Scale Turbo-Expander Development and its Performance Test Results", in Adv. in Cryo. Engr., Vol. 35 B, Plenum Press, NY (1990), 1005-1021.

14. C. K. Chan, E. Tward, and W. W. Burt, "Overview of Cryocooler Technologies for ... Sensors", in Adv. in Cryo. Engr., Vol. 35 B, Plenum Press, NY (1990), 1239-1250.

15. F. D. Doty et al, "An Ultra-Compact, Laminar-Flow Cryogenic Heat Exchanger", in Adv. in Cryo. Engr., Vol. 37 A, Plenum Press, NY (1992), 233-240.

16. F. D. Doty, B. L. Miller, G. S. Hosford, D. G. Wilson, W. Huanbo, and J. D. Jones, "High Efficiency Microturbine Technology", Proceedings, IECEC-91, 2 (1991), 436-442.

17. F. D. Doty, J. B. Spitzmesser, and D. G. Wilson, "High Temperature NMR Sample Spinner", U.S. Pat \# 5,202,633, 1993. 
18. David Gordon Wilson, The Design of High Efficiency Turbomachinery and Gas Turbines, MIT Press, Cambridge, 1984.

19. H. Kawamura, "Turbocharger for Internal Combustion Engines", U. S. Pat \# 4,769,993, 1988.

20. T. M. Jahns, "Fault-Tolerant Switched Reluctance Machine", U. S. Pat \# 4,896,088, 1990.

21. T. Hashimoto et al, "Recent Progress on Rare Earth Magnetic Regenerator Materials", in Adv. in Cryo. Engr., Vol. 37 B, Plenum Press, NY (1992) 859-865.

22. F. D. Doty, G. S. Hosford, J. B. Spitzmesser, and J. D. Jones, "The Microtube Strip Heat Exchanger", Heat Transfer Engr.,12, 3 (1991) 31-41.

23. F. D. Doty, "Microtube-Strip (MTS) Heat Exchanger", U. S. Pat \# 4,676,305, 1987.

24. N. Saji, S. Nagai, H. Asakura, Y. Kaneko, "Design of Oilfree All Turbo-type Helium Refrigerator", Cryogenics, Vol 34, ICEC Supp., (1994), 115-118.

25. S. A. Collins, A. H. von Flotow, and J. D. Paduano, "Analogue adaptive vibration cancellation system for Stirling cryocoolers", Cryogenics, Vol. 34, 5, Butterworth, 1994, 399-406.

26. W. L . Swift, W. E. Nutt, and H. Sixsmith, "A Helium Regenerative Compressor", in Adv. in Cryo. Engr., Vol. 39 B, Plenum Press, NY (1994), 901-908.

27. F. D. Doty and J. D. Jones, "A New Look at the Closed Brayton Cycle", Proceedings IECEC-90, Vol. 2, 1990, Am. Inst. Chem. Engr., NY, 166-172.

28. Photonics Design Handbook, 42nd Edition, 1996, Vol. 3, Laurin Publishing, Pittsfield, MA.

29. R. A. Ackermann, "Cryorefrigerator Evaluation for Future MRI Applications", Proceedings of the Sixth International Cryocoolers Conference, Plymouth, Mass. (1991) 3-16.

30. R. Triebe et al, "A User-Friendly System for the Routine Application of Cryogenic NMR Probes: Technology and Results", poster P201, ENC, Orlando, FL, 1999.

31. Vibration Solutions, 1997, Technical Manufacturing Corp., www.techmfg.com, Peabody, MA.

32. F. D. Doty et al, "A High-Pressure NMR Cell for Biochemical Applications", SBIR Phase I

Final Report, NIH Grant \# DMI-9560271, 1996.

33. G. Strumolo and V. Babu, "New Directions in Computational Aerodynamics," Physics World, 8/1997, 45-49.

34. F. D. Doty, "Double Rotation NMR Sample Spinner", U. S. Pat \# 5,325,059, 1994.

35. F. D. Doty, G. Entzminger, and Y. A. Yang, "Magnetism in HR NMR Probe Design: Part I", Concepts in Magn. Reson. (1998) 10:3, 133-156.

36. F. D. Doty et al, "High-Performance Class AB Pulse Amplifiers for MRI", NIH \#44-CM-77804, SBIR Phase II Final Report, 1989.

37. F. D. Doty and James K. Wilcher, "Crescent Gradient Coils", U. S. Pat \# 5,554,929, 1996. 